\title{
Developments in Minimally Invasive Surgical Options for Vertebral Pain: Basivertebral Nerve Ablation - A Narrative Review
}

\author{
Vinicius Tieppo Francio (1D) \\ David Sherwood ${ }^{2}$ \\ Eric Twohey ${ }^{3}$ \\ Brandon Barndt ${ }^{4}$ \\ Robert Pagan-Rosado (iD ${ }^{5}$ \\ James Eubanks ${ }^{6}$ \\ Dawood Sayed (1D) 7
}

'Department of Rehabilitation Medicine, University of Kansas Medical Center, Kansas City, KS, USA; '2Department of Physical Medicine and Rehabilitation, Vanderbilt University Medical Center, Nashville, TN, USA; ${ }^{3}$ Gundersen Health System Transitional, La Crosse, WI, USA; ${ }^{4}$ Department of Physical Medicine and Rehabilitation, Temple University and Moss Rehabilitation, Philadelphia, PA, USA; ${ }^{5}$ Department of Physical Medicine and Rehabilitation, Mayo Clinic, Rochester, MN, USA; ${ }^{6}$ Department of Physical Medicine and Rehabilitation, University of Pittsburgh Medical Center, Pittsburgh, PA, USA; ${ }^{7}$ Department of Anesthesiology, The University of Kansas Medical Center, Kansas City, KS, USA
Correspondence: Dawood Sayed Department of Anesthesiology, The University of Kansas Medical Center, 390I Rainbow Blvd, Mailstop 1034,

Kansas City, KS, 66103, USA

Tel + | 9|3-588-552|

$\mathrm{Fax}+1$ 9/3-588-3365

Email dsayed@kumc.edu

\begin{abstract}
Historically, intervertebral disc degeneration has been the etiological target of chronic low back pain; however, disc degeneration is not necessarily directly associated with pain, and many other anatomical structures are potential etiologies. The vertebral endplates have been postulated to be a source of vertebral pain, where these endplates become particularly susceptible to increased expression of nociceptors and inflammatory proliferation carried by the basivertebral nerve (BVN), expressed on diagnostic imaging as Modic changes. This is useful diagnostic information that can help physicians to phenotype a subset of low back pain, which is known as vertebral pain, in order to directly target interventions, such as BVN ablation, to this significant pain generator. Therefore, this review describes the safety, efficacy, and the rationale behind the use of BVN ablation, a minimally invasive spinal intervention, for the treatment of vertebral pain. Our current literature review of available up-to-date publications utilizing BVN ablation in the treatment of vertebral pain suggests that there is limited, but moderate-quality evidence that this is an effective intervention for reduction of disability and improvement in function, at short- and long-term follow-up, in addition to limited moderate-quality evidence that BVN RFA is superior to conservative care for pain reduction, at least at 3-month follow-up. Our review concluded that there is a highly clinical and statistically significant treatment effect of BVN ablation for vertebral pain with clinically meaningful benefits in pain reduction, functional improvements, opioid dose reduction, and improved quality of life. There were no reported devicerelated patient deaths or serious AEs based on the available literature. BVN ablation is a safe, well-tolerated and clinically beneficial intervention for vertebral pain, when proper patient selection and surgical/procedural techniques are applied.
\end{abstract}

Keywords: basivertebral nerve, ablation, neurotomy, vertebrogenic, vertebral pain, Modic changes

\section{Introduction}

Low back pain (LBP) has been consistently described as the most expensive occupational disorder in the United States, as well as the leading cause of disability worldwide. ${ }^{1-4}$ Chronic low back pain (CLBP), defined as axial low back pain which persists beyond three months, affects approximately 30 million people in the United States and costs the healthcare system 90 billion dollars per year. ${ }^{1,5-9}$ CLBP is the most common cause of activity limitation in individuals younger than 45 years of age, ${ }^{9}$ and it is the leading cause of absenteeism for persons between 30 and 64 years old, ${ }^{10}$ resulting in an estimated 100 million workdays lost annually. Additionally, 
CLBP has a well-known association with chronic opioid therapy, with an estimated $61 \%$ of affected patients receiving at least one prescription per year. ${ }^{10,11}$

Due to challenges with specificity in CLBP diagnosis, nearly $85 \%$ of individuals are given a diagnosis with a non-specific etiology. ${ }^{10}$ However, whether $85 \%$ of CLBP is truly non-specific has been successfully debated elsewhere but is beyond the scope of this manuscript. Importantly, the frequency of a non-specific CLBP diagnosis is one reason why many interventions exist (epidural injections, facet injection, spinal cord stimulators, vertebral augmentation, etc), each with varying levels of efficacy given the poor specificity of the diagnosis. Ideally, spine care would be best served by taking a page from the precision medicine playbook and aim to pursue the right treatment, for the right patient, at the right time, and directed to the correct anatomical target.

Historically, intervertebral disc (IVD) has been the etiological target of CLBP. However, disc degeneration is not associated with pain and is a non-pathologic process, which occurs asymptomatically and naturally with aging. Many other anatomical structures may be pain generators though, including internal disc disruption (IDD), zygapophyseal facet joints, sacroiliac joints, ligamentous structures, and spinal nerve roots. $^{1,10-13}$

The vertebral endplates, together with the IVD, act as a functional spinal unit, and they have been postulated to be a source of vertebrogenic pain, where these endplates become particularly susceptible to increased expression of nociceptors and inflammatory proliferation carried by the basivertebral nerve (BVN), a branch of the sinuvertebral nerve (SNV). 1,10,13,14 Vertebral endplates demonstrating Modic changes (MCs) on magnetic resonance imaging (MRI) tend to correlate with CLBP of vertebrogenic etiology (Figures 1 and 2). ${ }^{1,15-17}$ The presence of MCs on an MRI is useful diagnostic information that can help physicians to phenotype a subset of CLBP, which is vertebrogenic pain, in order to directly target interventions, such as basivertebral nerve ablation (BVNA), to this pain generator. ${ }^{1,17}$

BVNA is a minimally invasive spinal procedure that has recently gained attention to treat vertebral endplate pain.
A

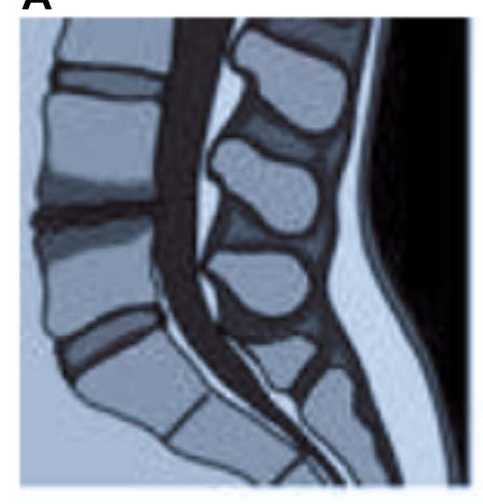

D

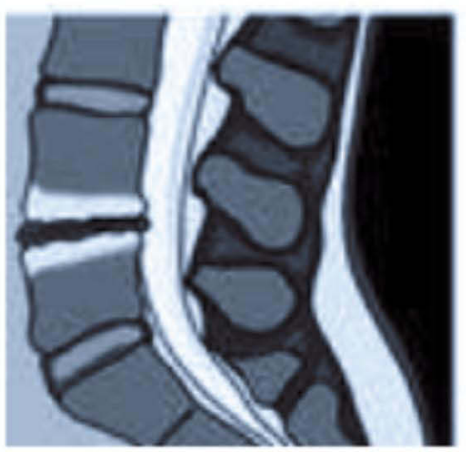

B

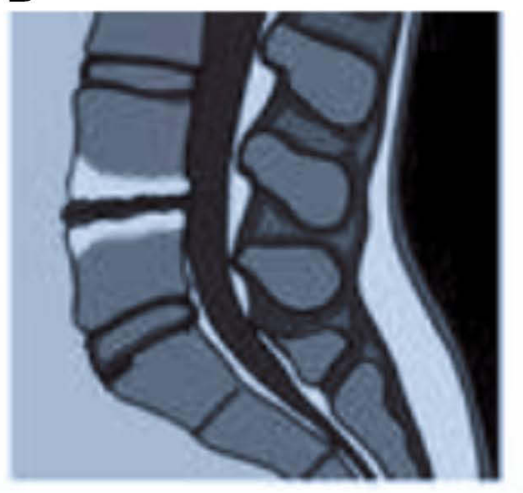

E

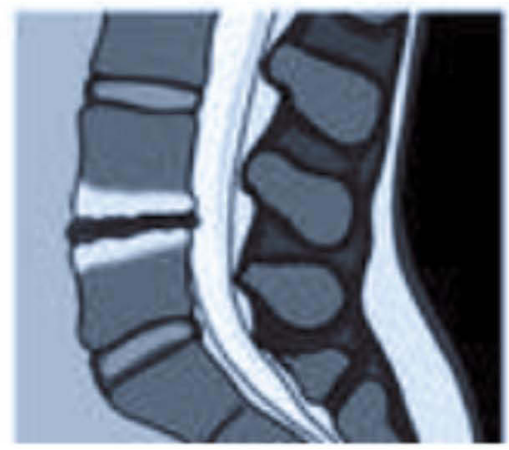

C

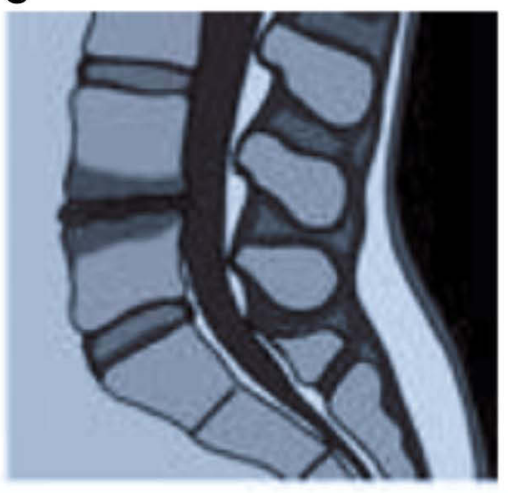

F

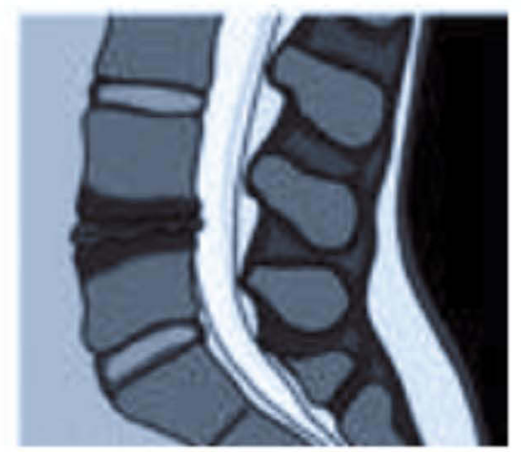

Figure I Schematic diagram demonstrating signal intensity changes in Modic patterns.

Notes: Types I Modic changes (A and D) intensity are low in TI MRI (A-C) and high in T2 MRI (D-F). Type 2 Modic changes (B and E) intensity are high in TI MRI and High in T2 MRI. Type 3 Modic changes (C and F) intensity are low in TI MRI and low in T2 MRI. Image courtesy of Relievant Medsystems Inc. 

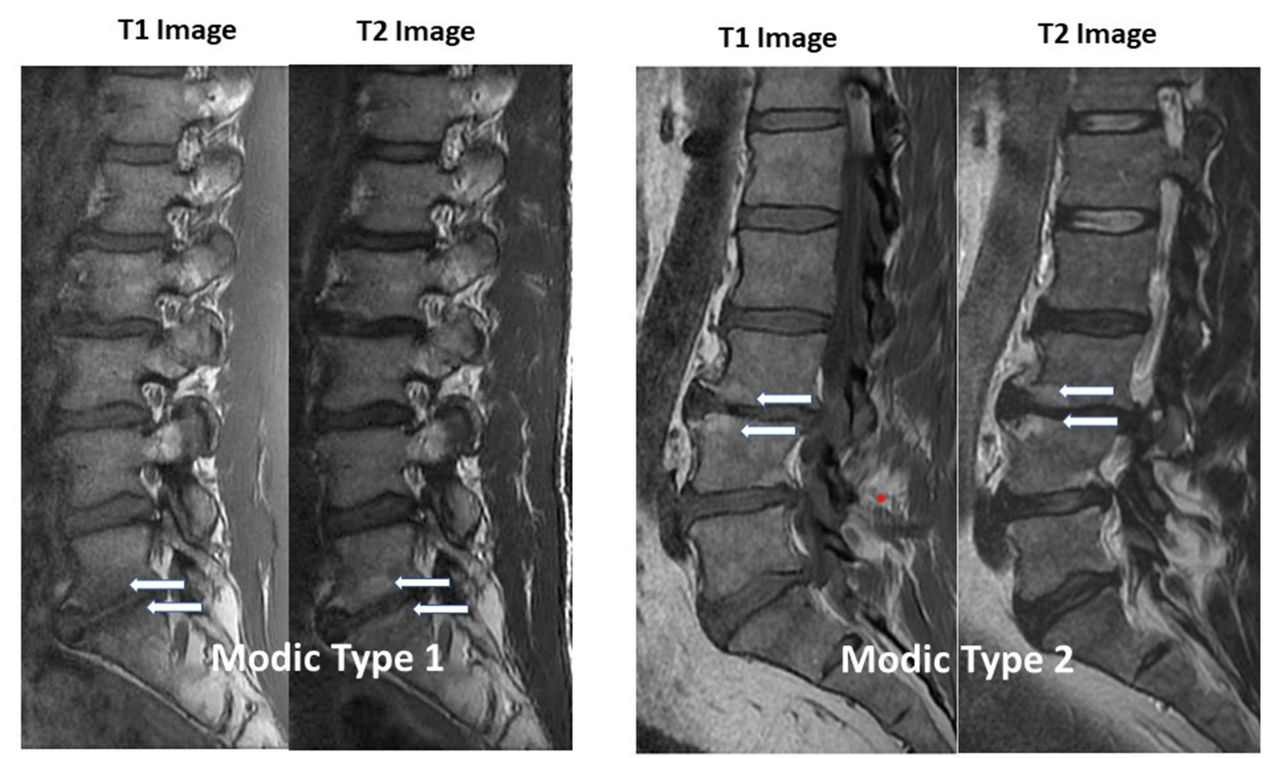

Figure 2 TI and T2 MRI images demonstrating Modic changes type I on the left and type 2 on the right.

Note: White arrows on the left are pointing to Modic changes type I, which represent vertebral endplates disruptions, fissuring and degeneration with active inflammation manifesting on MRI as hypodense or decrease signal intensity of fibrovascular intraooseous bone marrow edema. White arrows on the right are pointing to vertebral endplate Modic changes type 2, which manifest as hyperdense or increased signal intensity on MRI, representing fatty bone marrow infiltration/replacement. Image courtesy of Relievant Medsystems Inc.

The typical patient will have chronic axial low back pain without radicular symptoms and with radiological evidence of vertebral endplate Modic type 1 or type 2 changes on MRI. The studies upon which the rigid selection criteria for successful BVNA are described at length elsewhere in this manuscript. Damaged vertebral endplates produce high amounts of inflammatory cytokines leading to upregulation of nerve fibers and nociceptors, which correlates with axial back pain. BVNA serves as an option to treat damaged endplates by delivering a high-frequency alternating current that results in nerve denaturation and the interruption of pain transmission from vertebral endplates. ${ }^{1}$ This review describes the safety, efficacy, and the rationale behind the use of BVNA, a minimally invasive spinal intervention, for the treatment of vertebrogenic pain.

\section{Materials and Methods}

This study is a review of the literature aimed at appraising the available literature regarding the rationale, efficacy, and safety of basivertebral nerve ablation in the treatment of vertebrogenic pain. Data sources included PubMed, Medline, and Cochrane Library indexed manuscripts from the past two decades (March 2001-March 2021) with basivertebral nerve ablation (BVN RFA) keywords. Inclusion criteria included: human studies in the English language, such as randomized trials, meta-analyses, observational studies, and review articles. All included studies were independently appraised and collected by at least two separate authors in a standardized, unblinded fashion. Case reports, book chapters, commentaries, and letters to the editors were excluded. We summarized all relevant studies published to date regarding BVN RFA and provided a detailed summary of each study result, as well as a detailed discussion emphasizing each primary endpoint and study outcome. For all studies, data syntheses and analyses were performed with assessments of risk of bias, quality, and outcome measurements. We opted to utilize the preferred reporting items for systematic reviews and meta-analyses (PRISMA) flow diagram (Figure 3) in our methodology in order to reduce selection bias, by utilizing a standardized methodology and inclusion and exclusion criteria. Eighteen studies were found on the searched databases directly related to BVN RFA keywords. Of these, 3 were commentaries or letters to the editors and were excluded from our review, resulting in 15 available studies to review. Of these, 1 study was a systematic review, 1 study was a review of basivertebral anatomy, 1 study was a society guideline, and 2 studies were reviews regarding interventions for CLBP, citing BVN RFA. None of these studies included patient-related outcomes and therefore these are excluded from Table 1, but they were included in the discussion section of this 
manuscript. Ten studies reported primary outcomes with human subjects treated with BVN RFA. A summary of clinically and statistically significant findings from the 10 studies were compiled, and descriptive statistics are reported in Table 1 along with relevant comments comprehensively outlining the details of each study, statistical findings, sample size, follow-up time, and primary outcomes.

\section{Discussion}

\section{Rationale: Vertebral Pain and the Basivertebral Nerve}

Numerous studies have postulated that CLBP may have a vertebrogenic component rather than solely a discogenic one. There is growing evidence that damaged vertebral endplates can result in CLBP, with pain signals carried by the basivertebral nerve (BVN). Vertebrogenic CLBP with MCs has been shown to be clinically different than non-specific CLBP with reported greater frequency and longer duration of painful episodes, as well as worse outcomes with conservative treatment and surgery. ${ }^{11,16-21}$ As clinicians and researchers aim to optimize interventions to treat CLBP of vertebrogenic origin, it is important to understand the anatomical considerations of this pain generator.

During spinal column embryogenesis, sclerotomal cells develop from paraxial mesoderm and migrate towards the area surrounding the notochord and neural tube, and each of the 42-44 pairs of somites separate into cranial and caudal sections and a cell-free space. Eventually, the notochord becomes the nucleus pulposus, vertebral bodies develop from sclerotomal cells and the nucleus pulposus in between the two layers of sclerotomal cells, a caudal sclerotomal layer fuses with the adjacent cranial sclerotomal layer to form the vertebral body, and a neurovascular bundle enters to supply the vertebral body through the basivertebral foramen. ${ }^{22,23}$ The basivertebral foramen is located within the center of the posterior vertebral body and is the midline entry point for a branch of the sinuvertebral nerve, the basivertebral nerve. Once the BVN enters the vertebral body, it travels to a point about $30-55 \%$ of the vertebral body from posterior to anterior before it bifurcates cranially and caudally to innervate the vertebral endplates (Figures 4 and 5). The BVN bifurcation is the anatomical target for the ablative procedure,

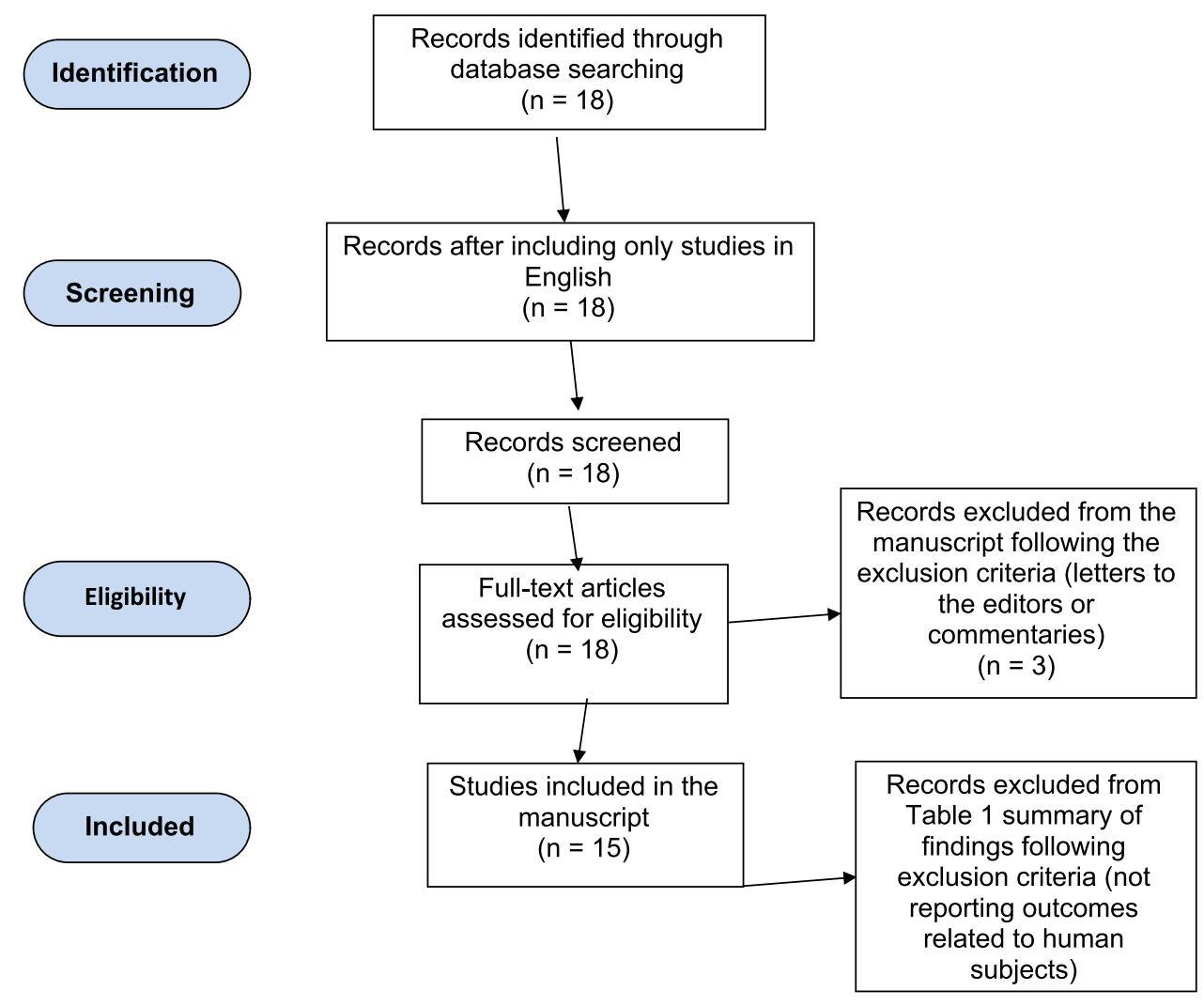

Figure 3 PRISMA flow chart methodology with identification, screening, eligibility and inclusion and exclusion process.

Note: Adapted from Page MJ, McKenzie JE, Bossuyt PM, et al. The PRISMA 2020 statement: an updated guideline for reporting systematic reviews. BMJ. 202I;372:n7I. ${ }^{4}$ 


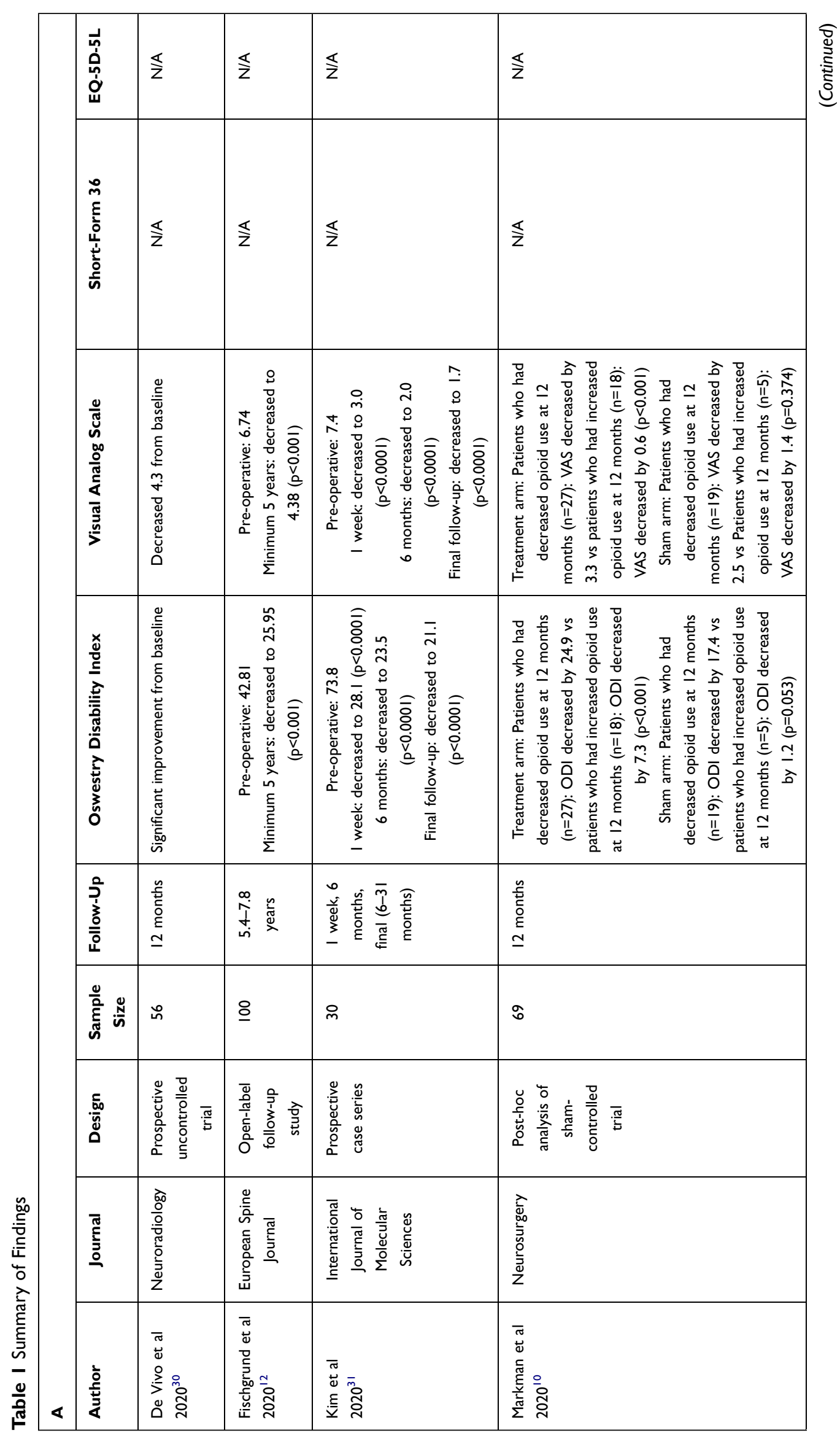




\begin{tabular}{|c|c|c|c|c|}
\hline & 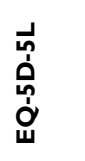 & 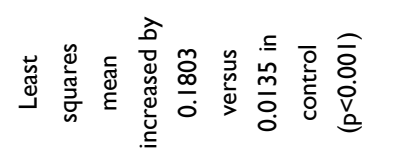 & 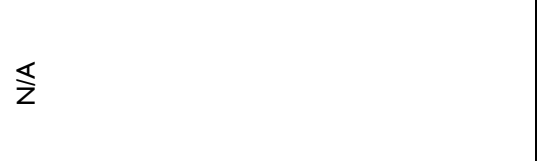 & 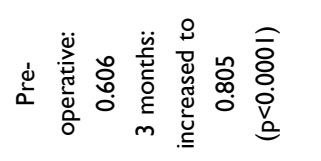 \\
\hline & 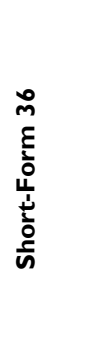 & 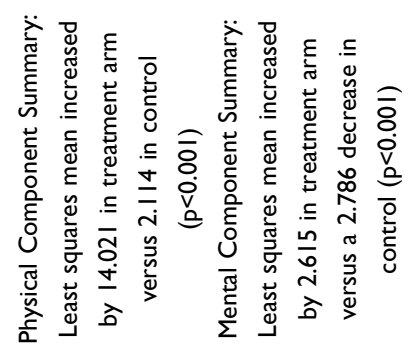 & 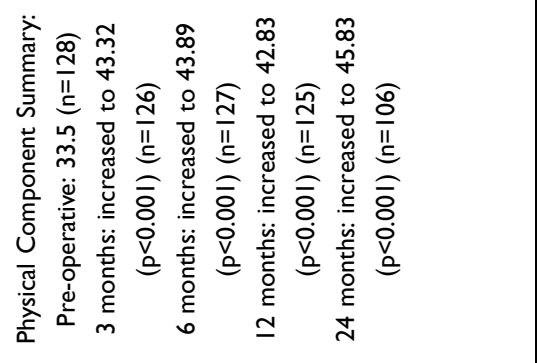 & 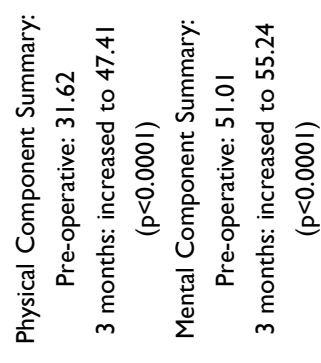 \\
\hline & 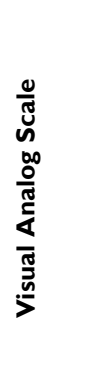 & 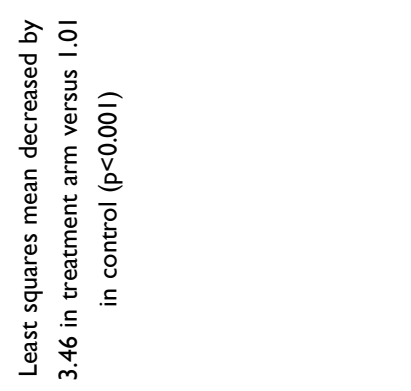 & 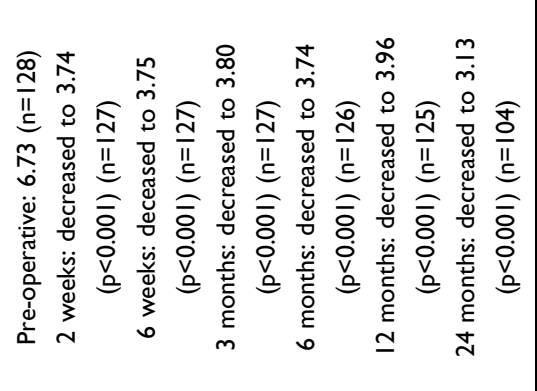 & 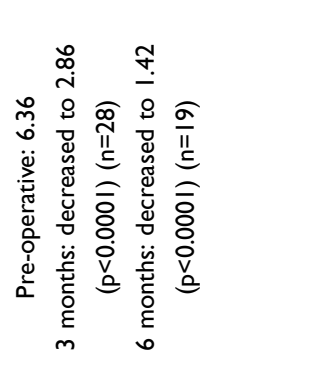 \\
\hline & 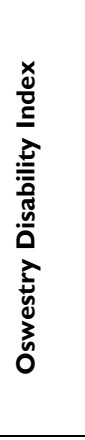 & 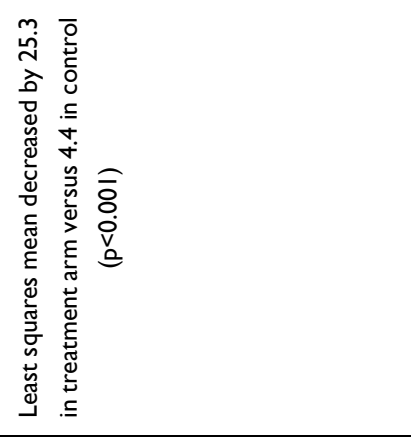 & 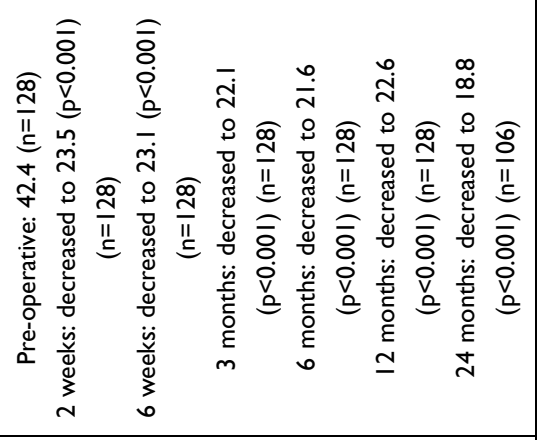 & 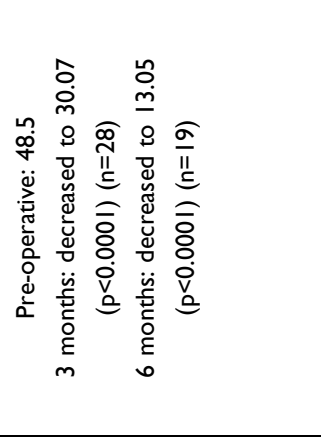 \\
\hline & 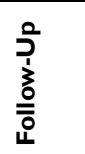 & 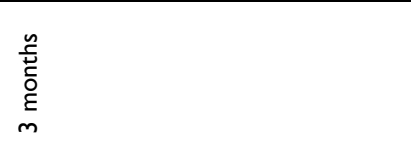 & 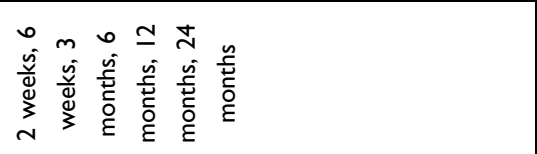 & 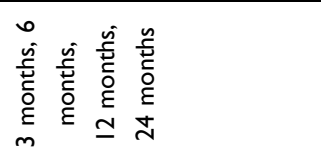 \\
\hline & $\frac{0}{\frac{0}{0}}$ & 므 & $\stackrel{\circ}{ }$ & $\stackrel{\infty}{\sim}$ \\
\hline & $\begin{array}{l}\frac{c}{.50} \\
\overline{\bar{\nu}} \\
\Delta\end{array}$ & 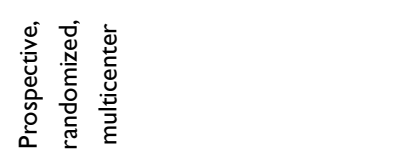 & 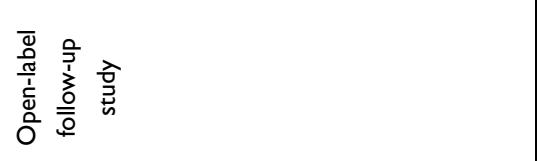 & 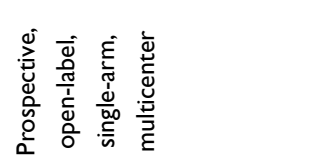 \\
\hline & 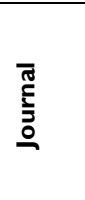 & 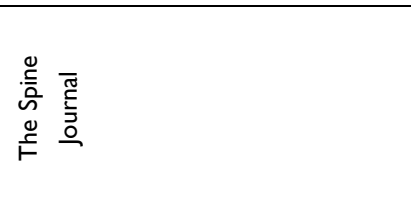 & 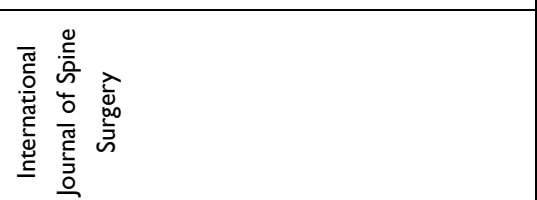 & 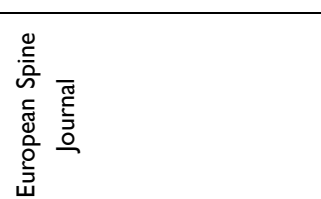 \\
\hline$\varangle$ & 嵩 & 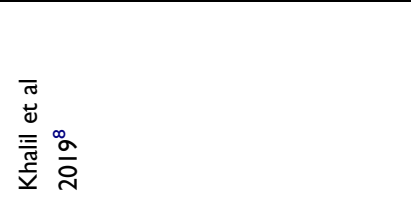 & 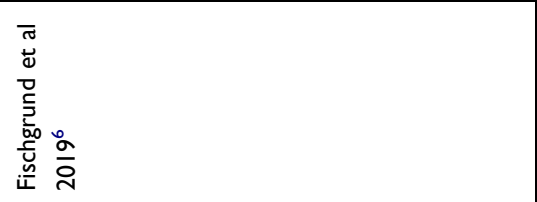 & 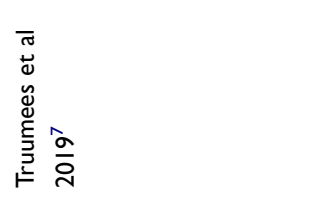 \\
\hline
\end{tabular}




\begin{tabular}{|c|c|c|}
\hline$\overleftarrow{Z}$ & 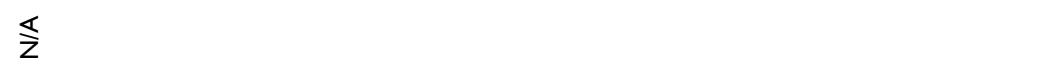 & $\overleftarrow{z}$ \\
\hline 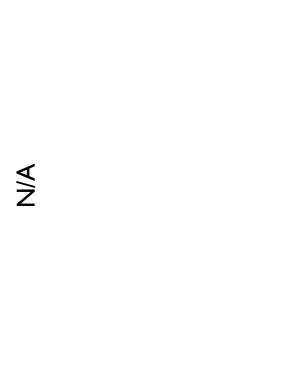 & 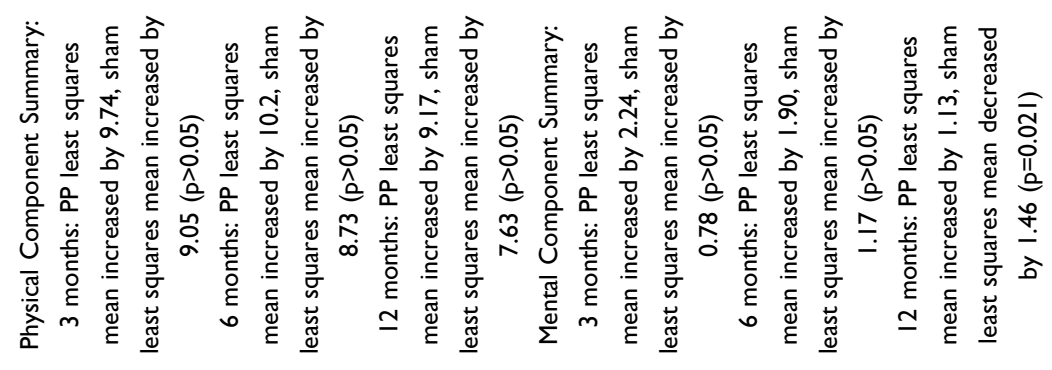 & 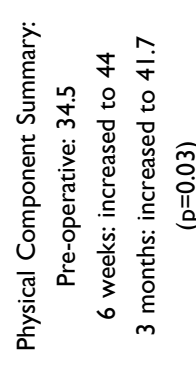 \\
\hline 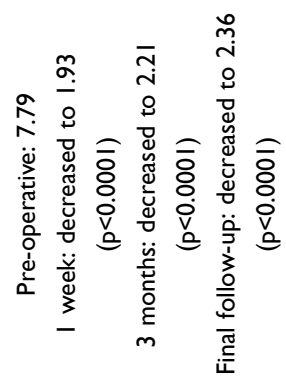 & 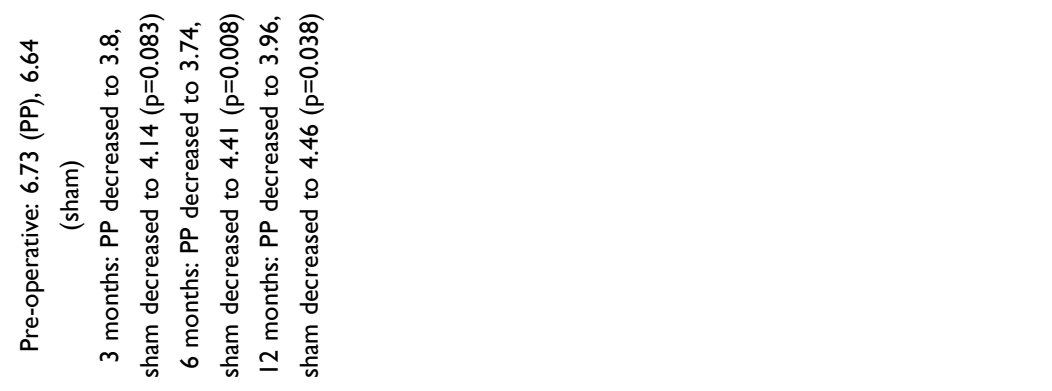 & 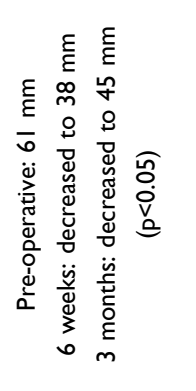 \\
\hline$\overleftarrow{z}$ & 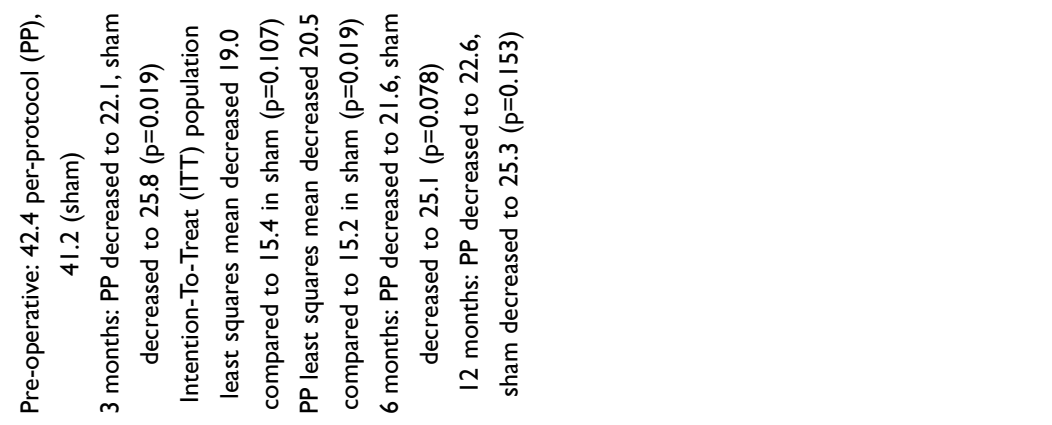 & 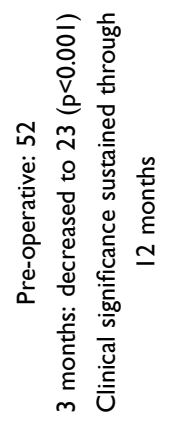 \\
\hline 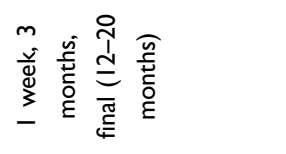 & 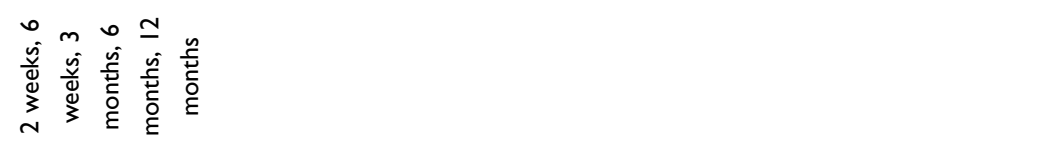 & 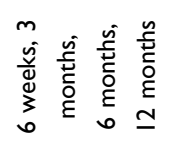 \\
\hline \pm & 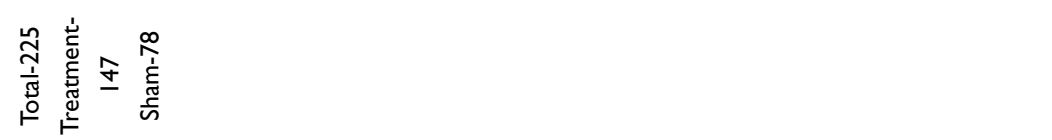 & $\stackrel{\circ}{\prime}$ \\
\hline 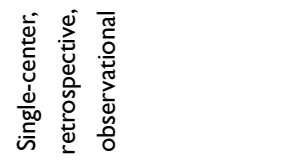 & 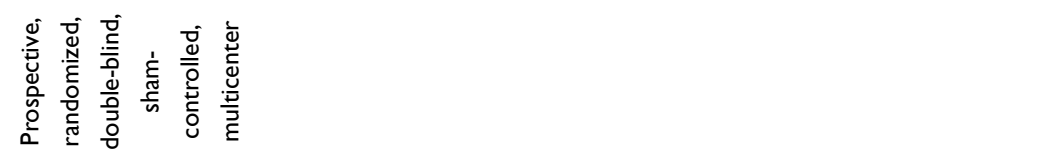 & 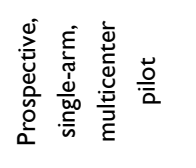 \\
\hline 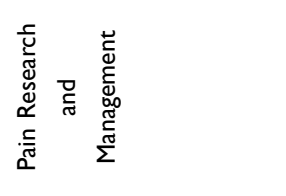 & 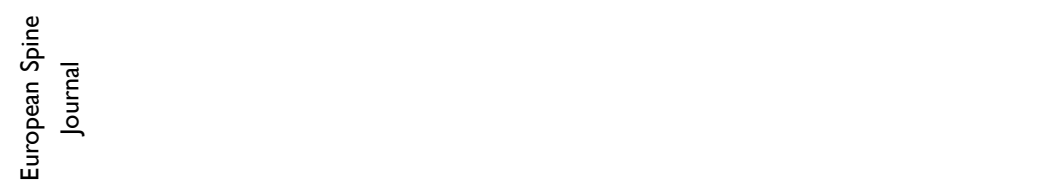 & 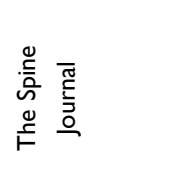 \\
\hline 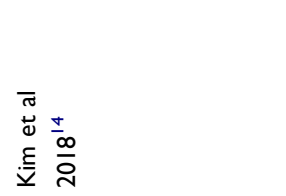 & 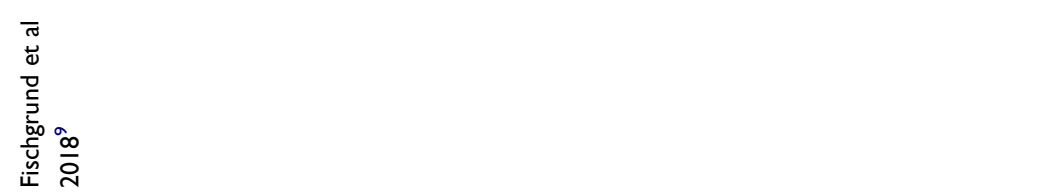 & 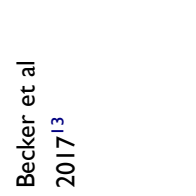 \\
\hline
\end{tabular}




\begin{tabular}{|c|c|c|c|c|c|}
\hline & 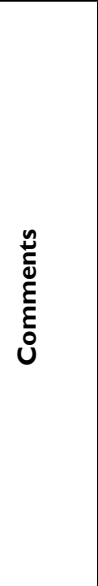 & 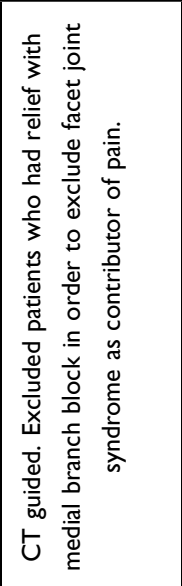 & 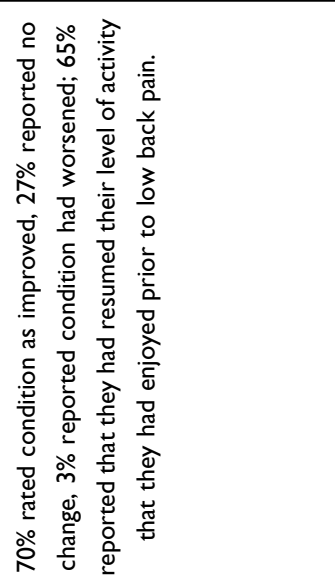 & 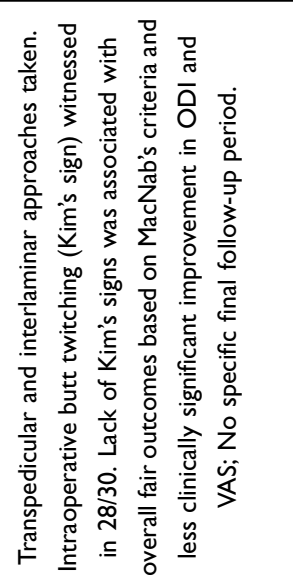 & 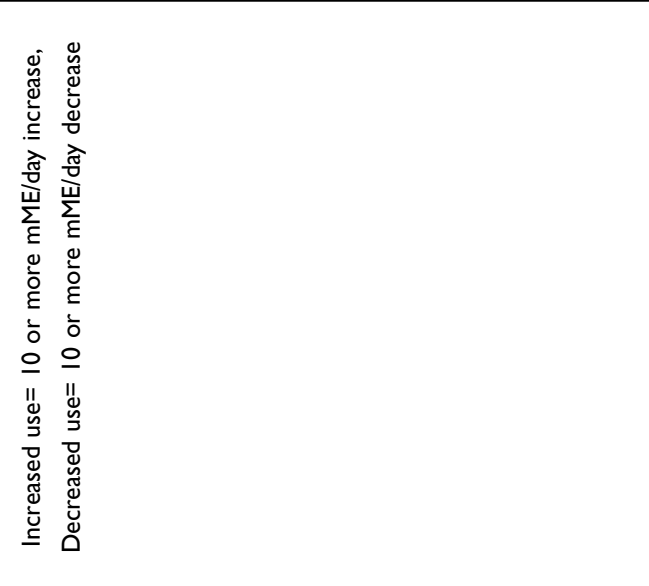 \\
\hline & 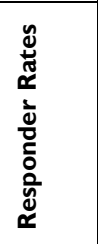 & 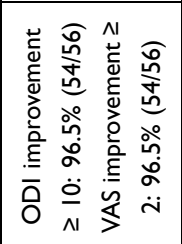 & 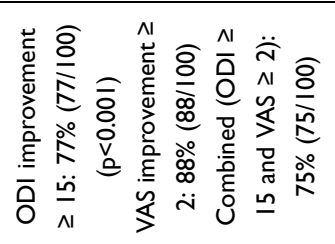 & $\overleftrightarrow{z}$ & 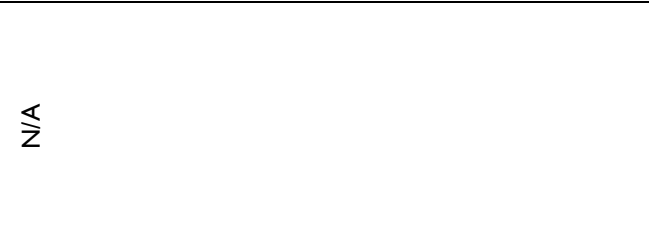 \\
\hline & 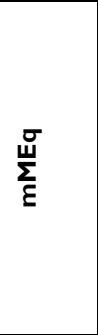 & 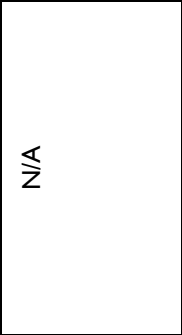 & 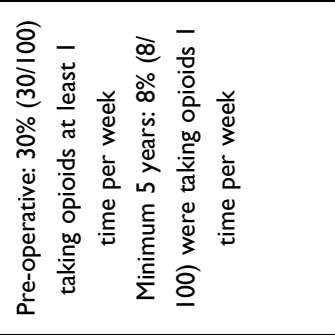 & 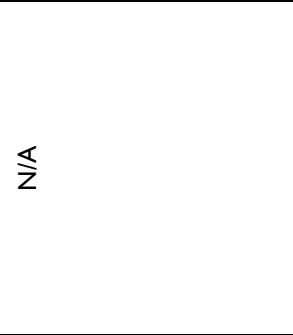 & 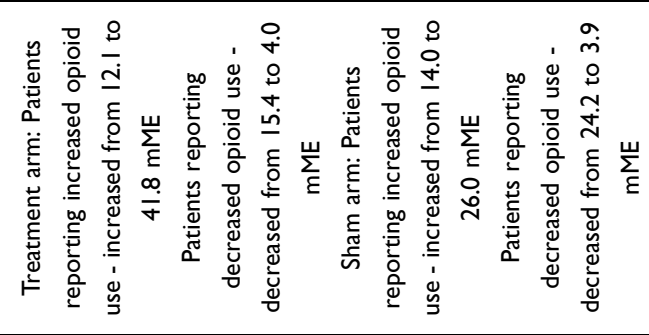 \\
\hline & 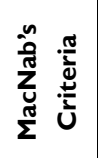 & $\stackrel{\nwarrow}{z}$ & 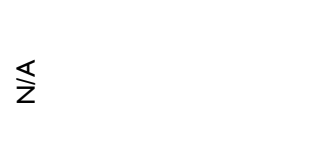 & 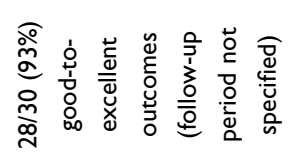 & $\overleftarrow{z}$ \\
\hline & 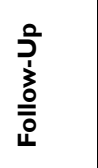 & 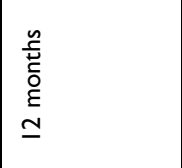 & 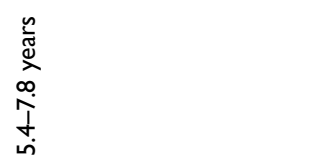 & 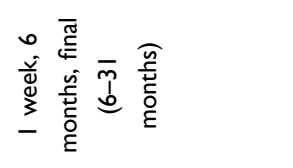 & 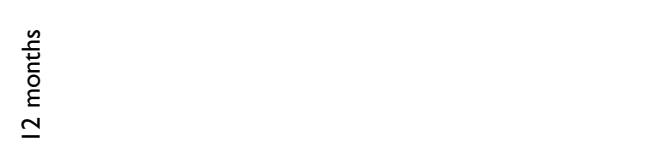 \\
\hline & 总 & மீ & 으 & 이 & o \\
\hline & 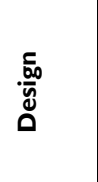 & 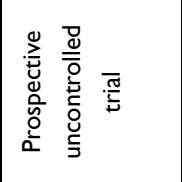 & 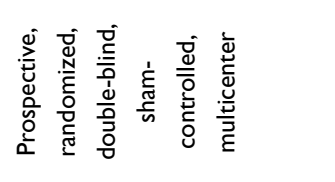 & 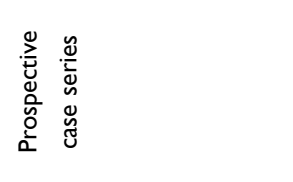 & 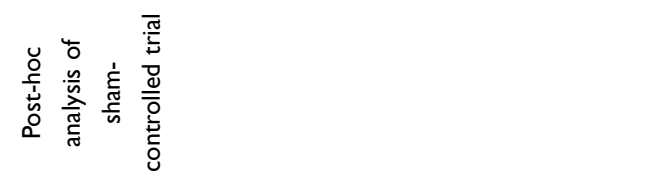 \\
\hline & 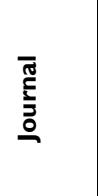 & 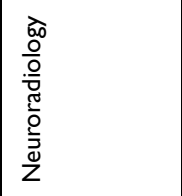 & 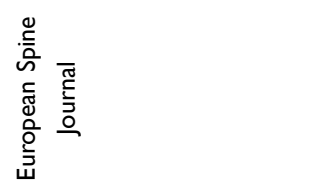 & 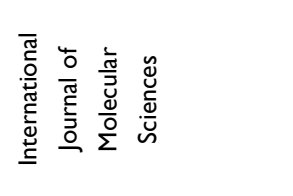 & 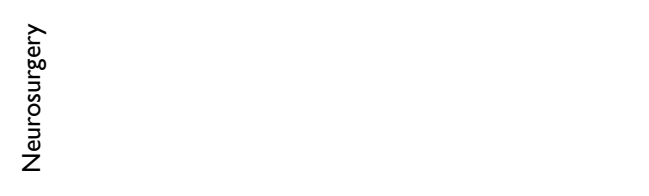 \\
\hline$\infty$ & 京 & 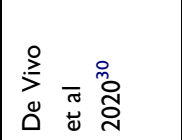 & 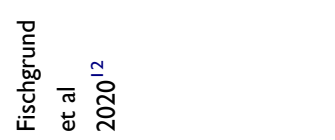 & 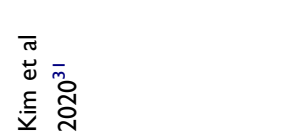 & 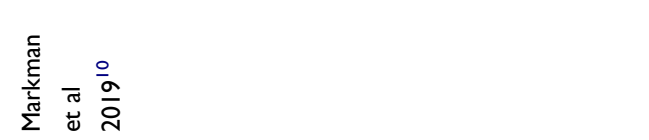 \\
\hline
\end{tabular}




\begin{tabular}{|c|c|}
\hline 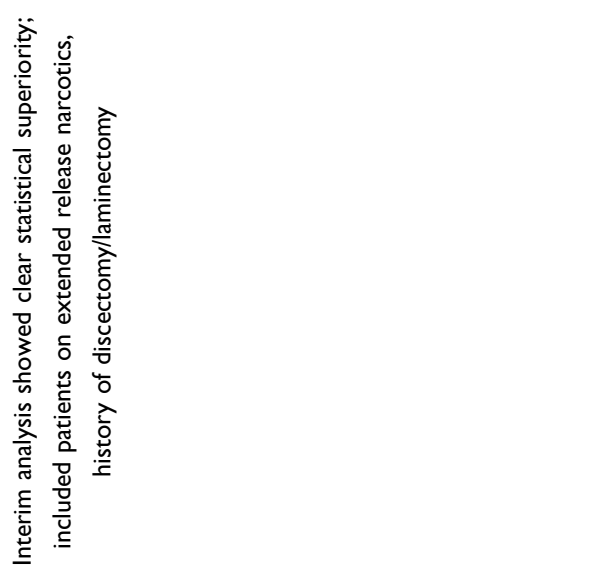 & 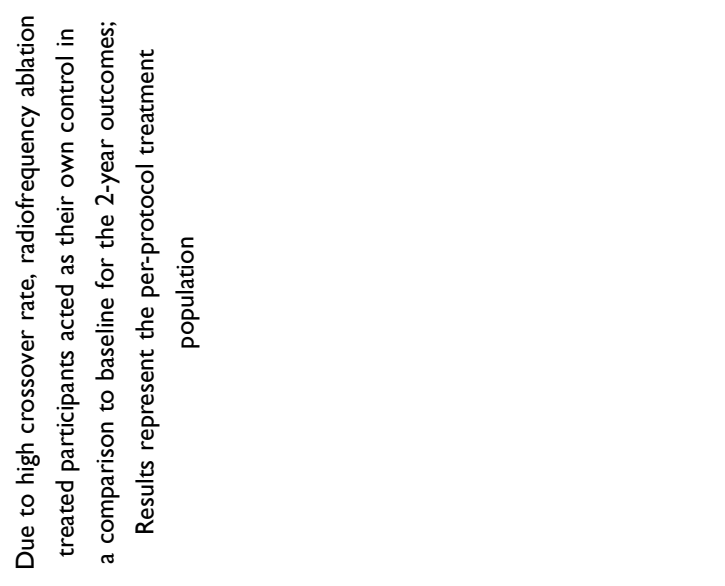 \\
\hline 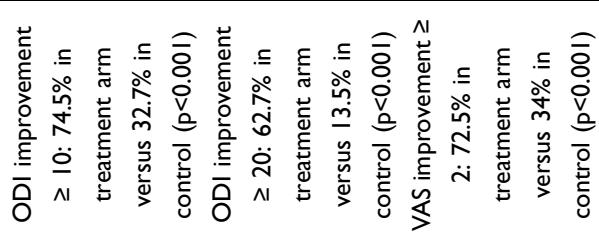 & 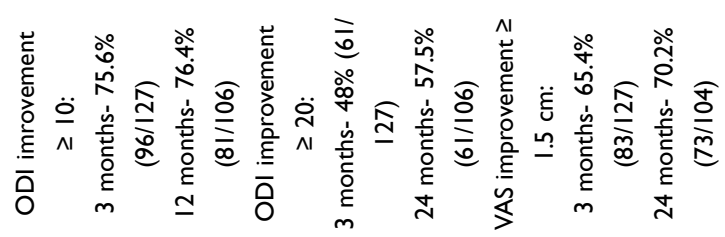 \\
\hline 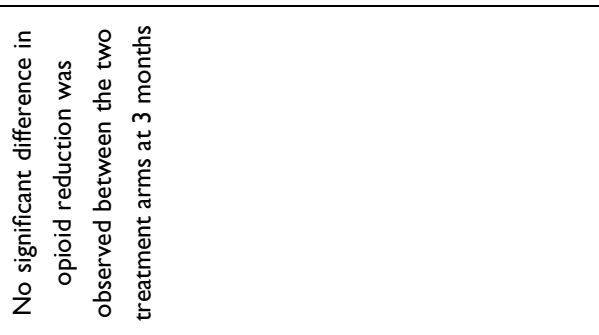 & $\S$ \\
\hline 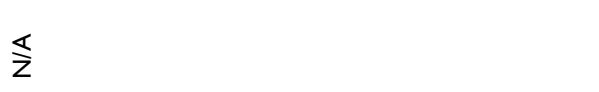 & 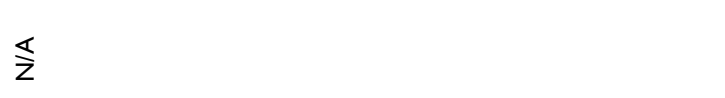 \\
\hline 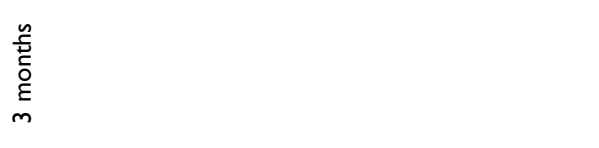 & 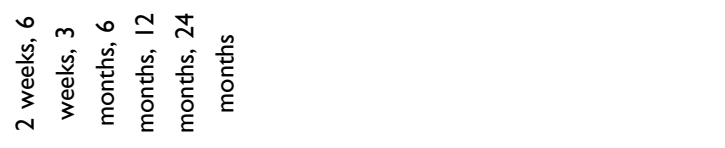 \\
\hline 壵 & ㅇ \\
\hline 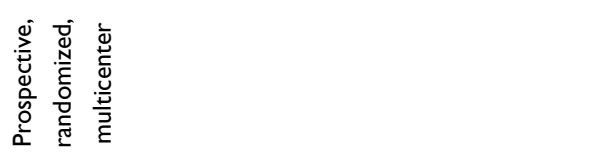 & 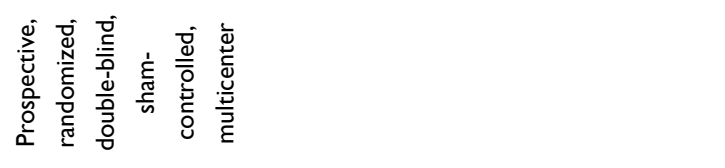 \\
\hline 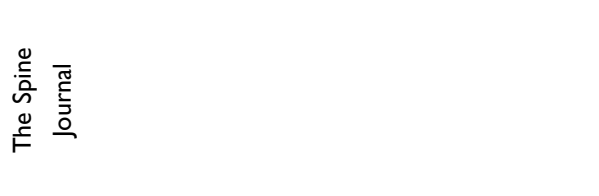 & 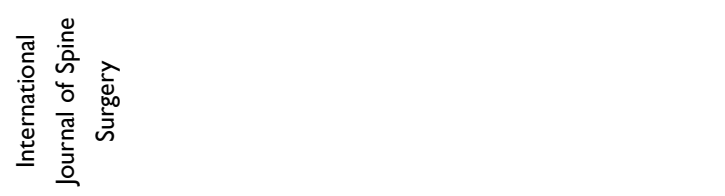 \\
\hline 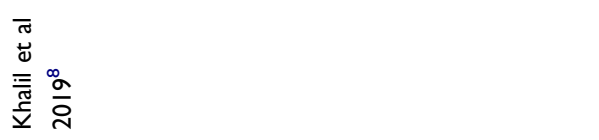 & 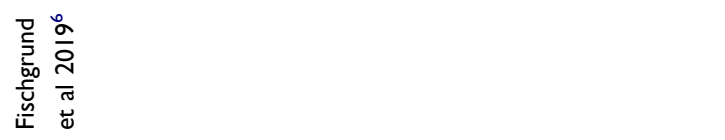 \\
\hline
\end{tabular}




\begin{tabular}{|c|c|c|c|c|c|}
\hline & 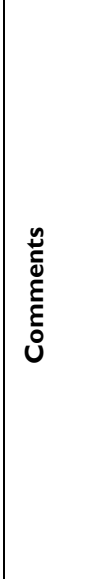 & 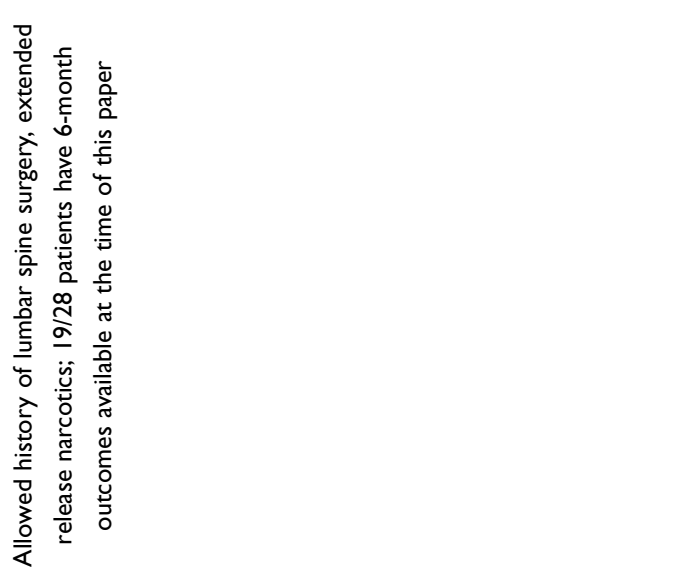 & 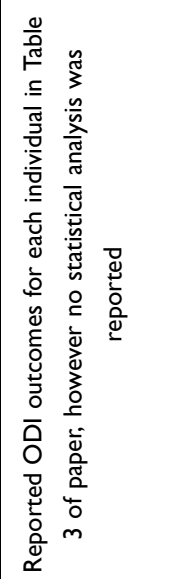 & 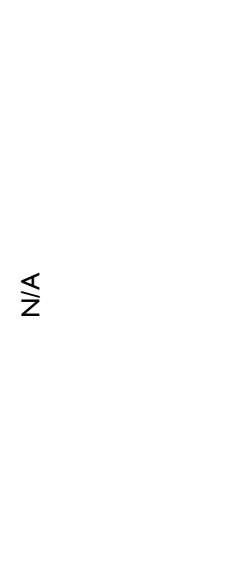 & 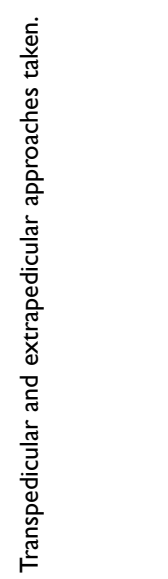 \\
\hline & 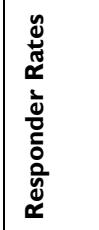 & 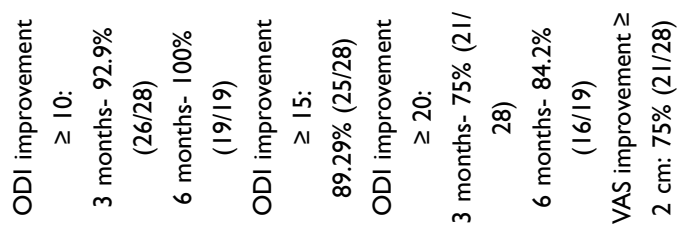 & 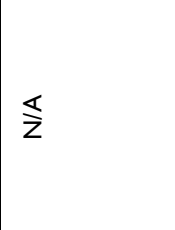 & 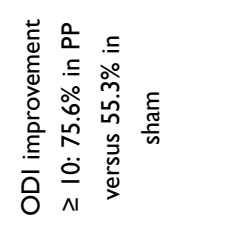 & 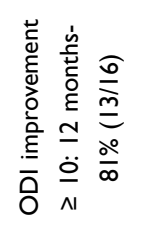 \\
\hline & $\sum_{\varepsilon}^{\mathbb{W}}$ & 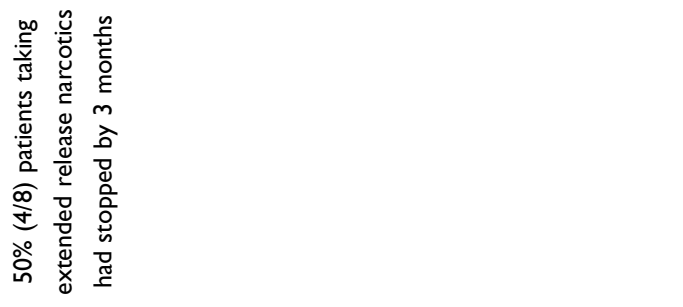 & $\leqslant$ & $\ll$ & $\$$ \\
\hline & 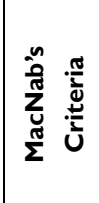 & 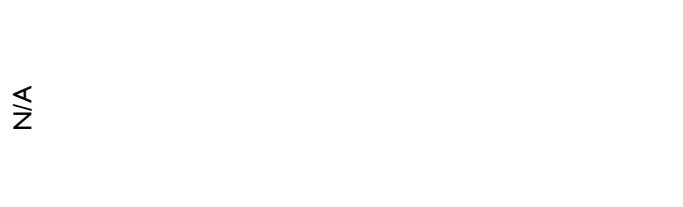 & 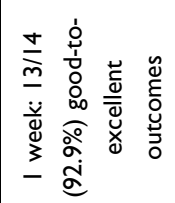 & $\widehat{\mathbb{z}}$ & 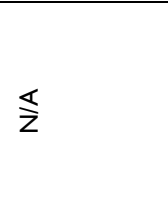 \\
\hline & 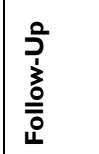 & 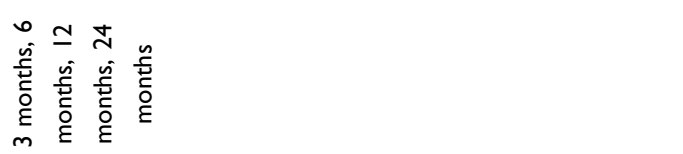 & 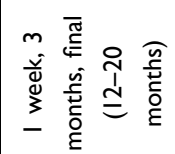 & 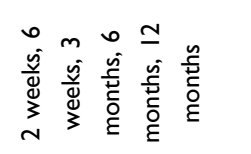 & 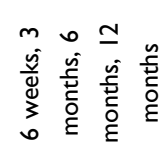 \\
\hline & 造 & $\stackrel{\infty}{\sim}$ & \pm & 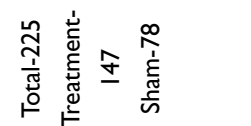 & $\underline{0}$ \\
\hline & $\begin{array}{l}\frac{.5}{.00} \\
\bar{\Delta} \\
0\end{array}$ & 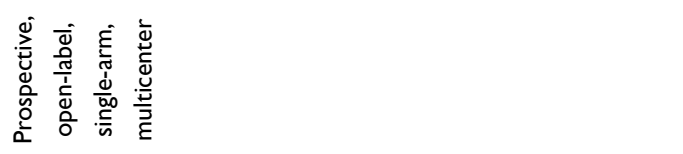 & 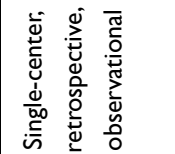 & 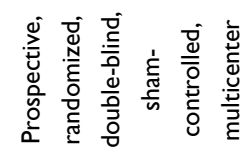 & 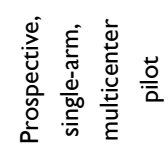 \\
\hline & 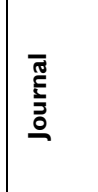 & 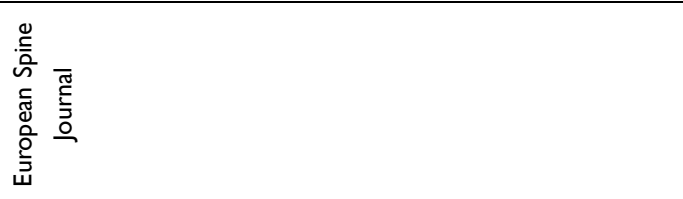 & 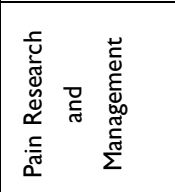 & 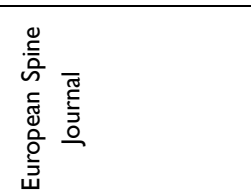 & 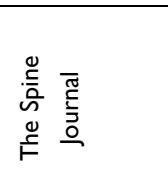 \\
\hline$\infty$ & 嵩 & 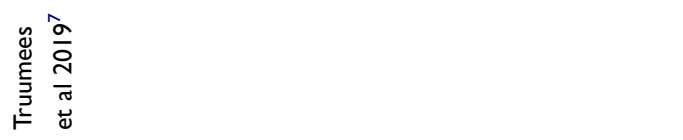 & 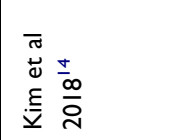 & 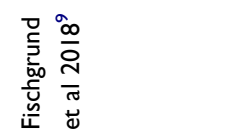 & 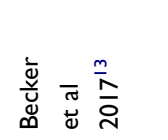 \\
\hline
\end{tabular}


since it is well known that the BVN is responsible for carrying nociceptive information from damaged vertebral endplates through substance $\mathrm{P}$ and calcitonin gene-related peptide (CGRP), which has been confirmed with PGP 9.5 positive staining, and is likely responsible for painful syndromes of vertebrogenic origin. ${ }^{16,19}$

Historically, other structures such as the intervertebral disc, facets, and ligaments have been studied as the source of pain in axial CLBP etiologies. Unfortunately, many interventions for the treatment of CLBP related to treating discogenic etiology have been unsuccessful. Hence, a shift towards other structures, such as vertebral endplates, has been proposed in recent human studies and successful outcomes for vertebrogenic-directed treatment have been consistently reported at both short and long-term followup with BVNA. Vertebral endplates are highly vascularized and innervated compared to the intervertebral disc and associated ligamentous structures, and the presence of MCs in MRIs are a successful predictor of pain of vertebrogenic nature, in addition to a thorough history and physical exam. Vertebral endplate MCs are indicative of intraosseous edema and inflammation and are directly correlated with the presence and severity of back pain, suggesting a phenotypic pattern not seen in any other CLBP etiology that is highly valuable to physicians and surgeons to optimize outcomes. ${ }^{24-27}$ The clinical presentation of vertebrogenic pain varies widely in the population, but it generally is described as a midline, deep, aching, burning pain of progressive nature, usually associated with an intermittent electrical shock sensation. There is no radicular expression, lower extremity weakness, nor sensory deficits and the neural tension sign and pain tends to be worse with spinal flexion, sitting, standing and with general physical activity, in contrast to extension. ${ }^{17,26,28,29}$ To provide additional insight on vertebrogenic pain symptomatology responsive to BVNA, aggregated data from over 320 clinical trial reported by Relievant Medsystems, patients revealed that $70 \%$ of patients indicated their pain location as midline on body diagrams and less than $6 \%$ reported pain below the mid-gluteal region.

In summary, CLBP is a highly prevalent and debilitating diagnosis with controversial management and limited successful outcome rates. Perhaps due to the anatomical complexities of the spinal unit with numerous potential painful sources, treatment options to date have produced variable outcomes. Recently, researchers have made progress focusing on direct target interventions in CLBP, particularly towards a vertebrogenic phenotype with

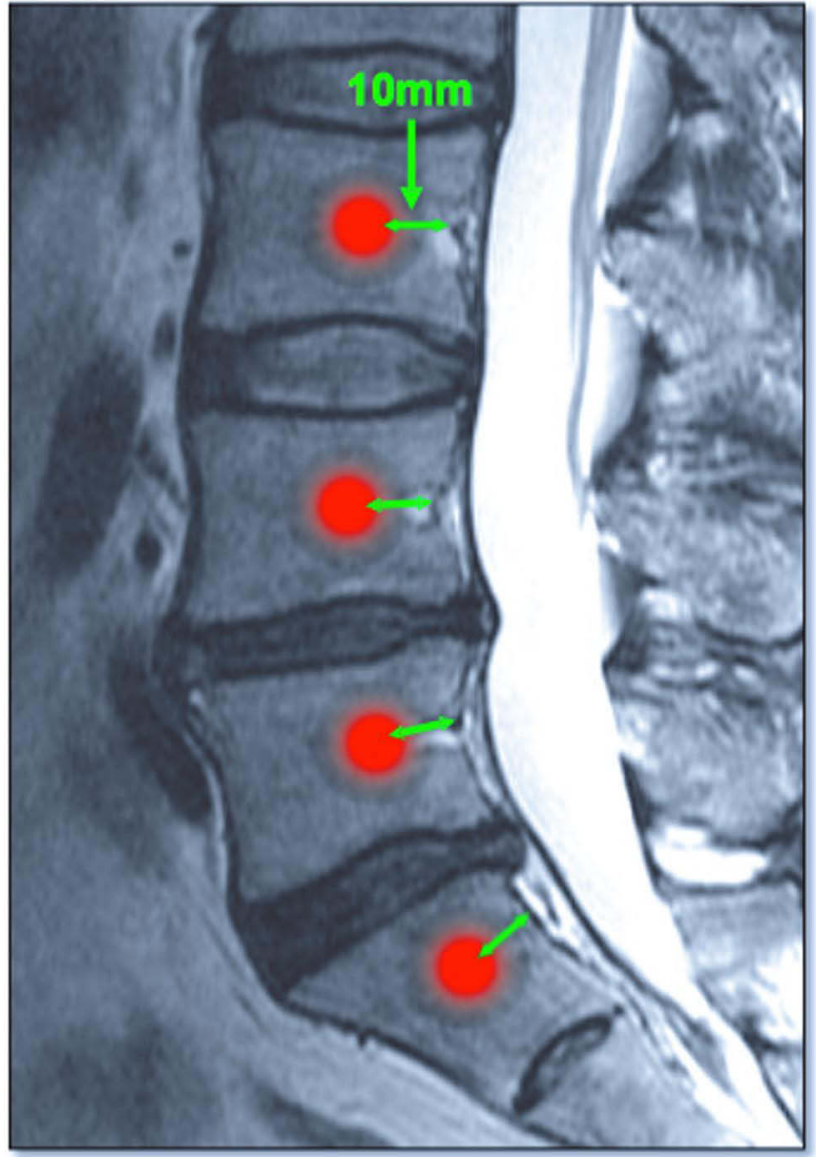

Figure 5 Magnetic Resonance Image (MRI) with green arrow demonstrating the location of the basivertebral nerve $10 \mathrm{~mm}$ posterior to anterior distance, site of the ablative procedure (red dot).

Note: Image courtesy of Relievant Medsystems Inc.

MCs. With the growing body of evidence supporting the BVN's role as a source of nociceptive pathway in low back pain, radiofrequency ablation of the BVN has emerged as a powerful minimally invasive procedure to treat vertebrogenic pain.

\section{Basivertebral Nerve Radiofrequency Ablation Procedure}

The procedure is performed by spine surgeons and interventional pain physicians in an outpatient setting with the patient sedated under conscious sedation or general anesthesia and placed in a prone position. Using fluoroscopic imaging or CT-guidance, the predetermined target level(s) location of entry is marked for a unilateral transpedicular approach. Previous studies have described a transforaminal endoscopic extrapedicular approach as well. $^{14}$

First, the skin is anesthetized, and a small incision is made with a scalpel. The introducer cannula is advanced 

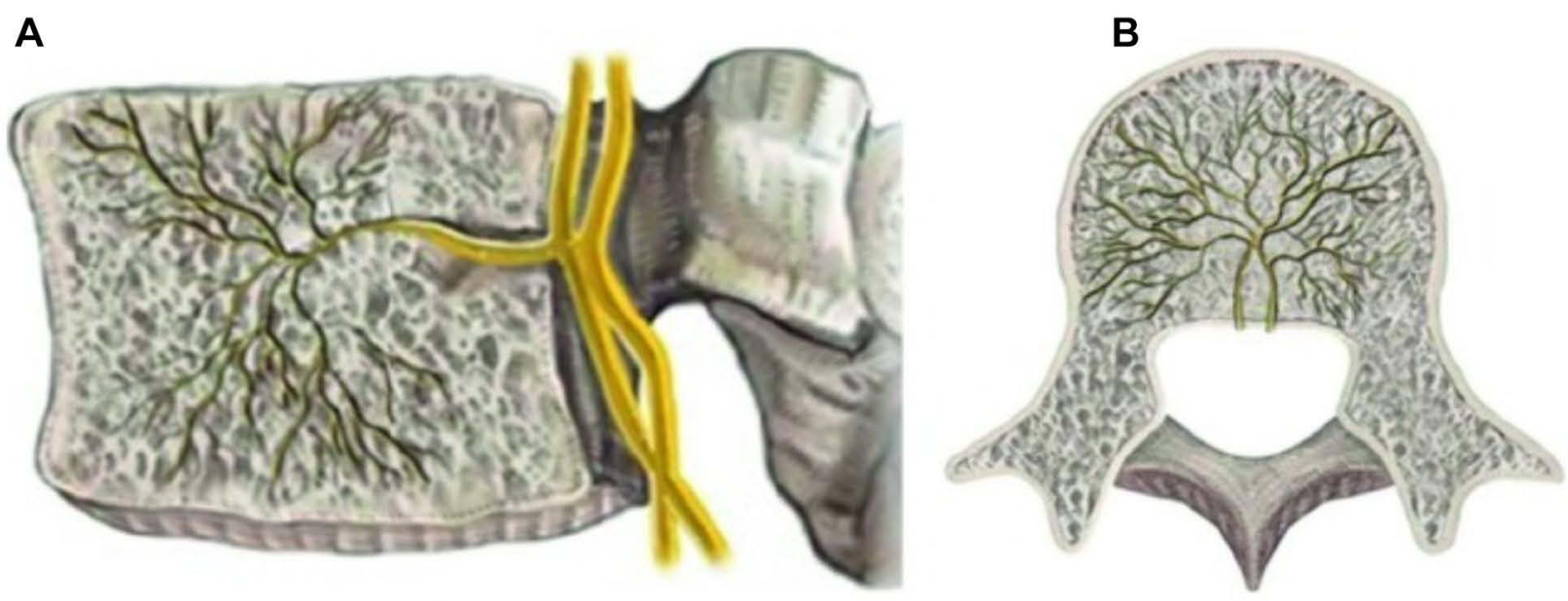

Figure 4 Sagittal (A) and axial views (B) of the sinuvertebral nerve as it enters the vertebral body through the basivertebral foramen, becoming the basivertebral nerve. Note: Reproduced from Kim HS, Adsul N, Yudoyono F, et al. Transforaminal Epiduroscopic Basivertebral Nerve Laser Ablation for Chronic Low Back Pain Associated with Modic Changes: A Preliminary Open-Label Study. Pain Res Manag. 2018;2018:6857983. ${ }^{14}$

through the pedicle starting at the superior lateral aspect until the posterior wall is breached. Positioning along the posterior aspect of the pedicle is confirmed with a biplanar visualization approach and the cannula is advanced superior lateral to inferior medial in order to obtain an optimal position for the cannula. A curved cannula then helps to create a channel to the basivertebral nerve terminus. The terminus can be found midline in the AP view and approximately $30-50 \%$ across the vertebral body width from the posterior wall. Preoperative MRI can be used to assist in identifying the patient's unique intraosseous site of bifurcation of the BVN. ${ }^{9,13,29}$ Of note, the BVN of the S1 vertebral body varies in anatomical location compared to the L4-5 based on the entry point. The L4-5 entry point is along the posterior border with a posterior-anterior path, and the $\mathrm{S} 1$ is along the lateral border with a lateral-medial path with the nerves clustering at the vertebral center. Once proper placement is confirmed, the curved cannula is removed and the radiofrequency probe is inserted into the created channel (Figures 6 and 7). A 10mm margin is established to ensure the safety of posterior structures, and the radiofrequency probe is inserted and activated to maintain a temperature of $85^{\circ} \mathrm{C}$ for 15 minutes with the goal of creating a $1 \mathrm{~cm}$ spherical lesion at the terminus. Once the radiofrequency lesion is created, the probe and cannula are removed, and the procedure is finalized by securing the puncture sites with steri-strips and pressure dressings. Sutures and staples are usually not necessary, and postoperative management follows the standard of care of other minimally invasive spinal interventions.

\section{Clinical Efficacy}

In this study, we discussed the available published literature regarding the clinical efficacy of BVNA for the treatment of vertebrogenic pain. The summary of relevant findings for all studies reviewed can be found in Table 1. Additionally, in Table 1 we provided critical appraisal of the current literature and further categorized the impacts of BVN RFA based on outcome measurements. Per the foundational studies discussed at length moving forward in this manuscript, BVN RFA may be successfully utilized in patients with axial chronic low back pain of vertebral etiology of at least 6-months duration, who have failed conservative treatment for at least 6-months, have radiological evidence of MCs type 1 or type 2 on MRI between the L3-S1 spinal segments while also fulfilling a number of other criteria discussed at length later, namely the absence of radicular symptoms, no history of previous lumbar surgery, and no osteoporosis, etc. ${ }^{1,28,29}$

The majority of studies reviewed reported functional outcomes with the Oswestry Disability Index (ODI) and pain assessment utilizing the visual analog scale (VAS). Quality of life measurements were reported utilizing the short form 36 (SF-36) and/or the EQ-5D-5L questionnaire, patient satisfaction was analyzed utilizing the MacNab's criteria, and opioid utilization was also reported as an outcome measurement. 
A

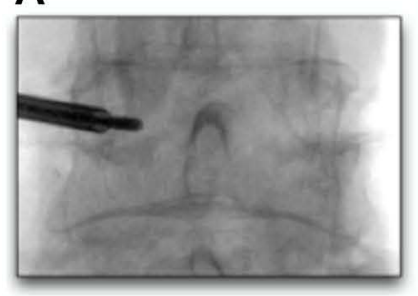

E

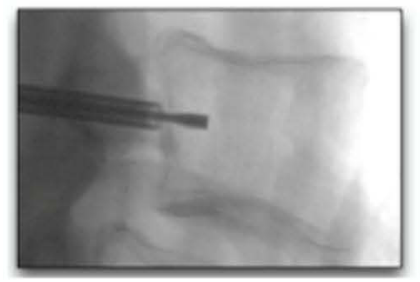

B

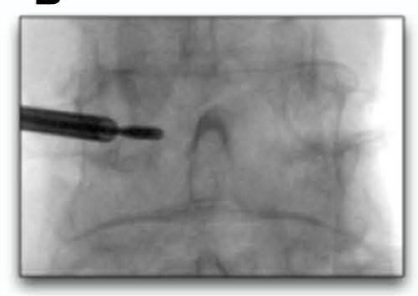

$\mathbf{F}$

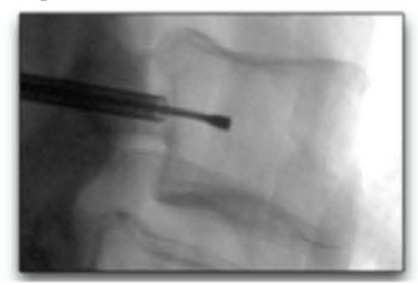

C

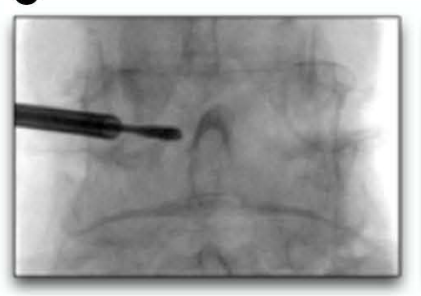

G

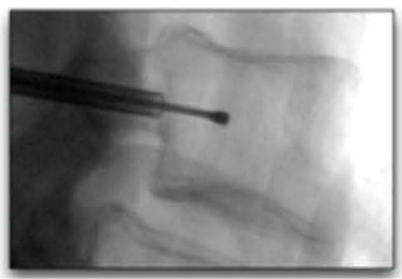

D

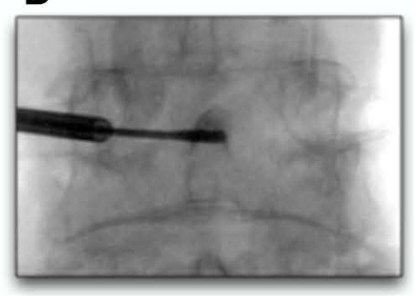

H

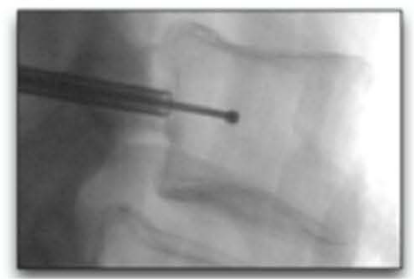

Figure 6 AP (A-D) and Lateral (E-H) fluoroscopy views of curved stylet advancement towards the ideal location between the $25-40 \%$ midline, between the anterior and posterior vertebral walls.

Note: Image courtesy of Relievant Medsystems Inc.

A

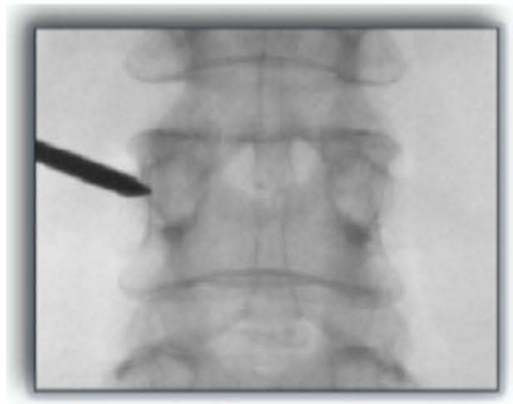

D

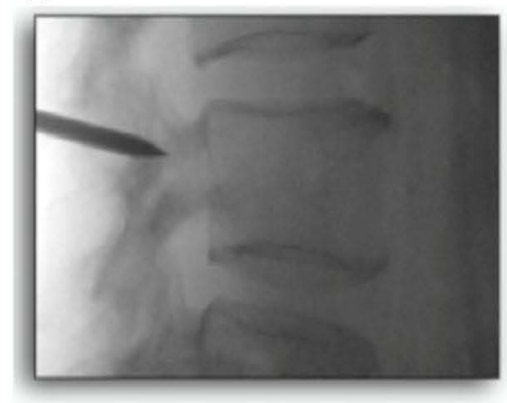

B

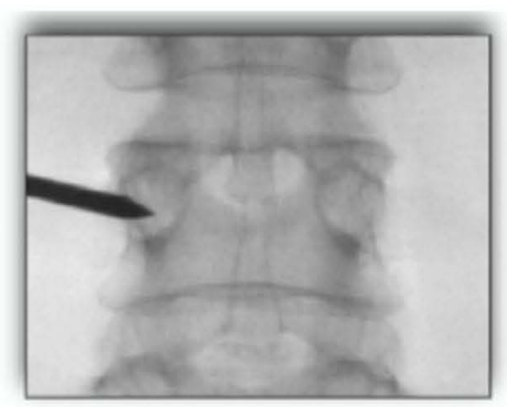

E

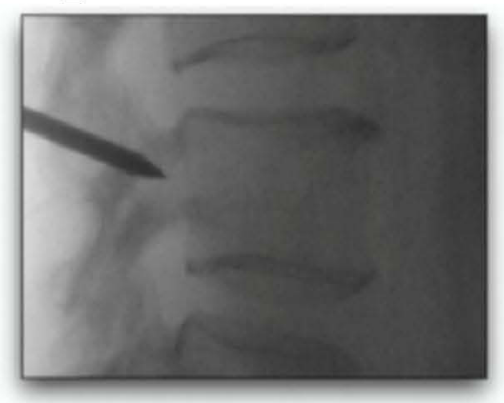

C

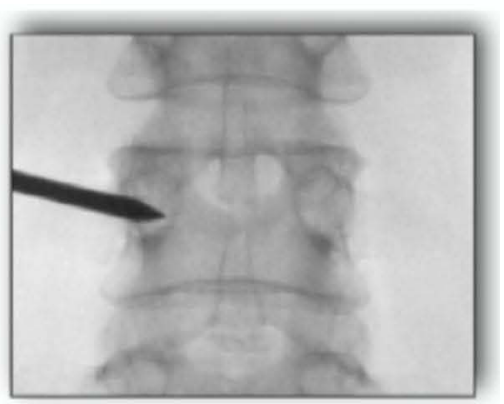

$\mathbf{F}$

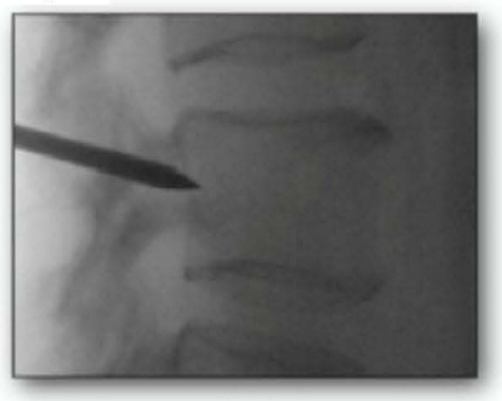

Figure 7 AP (A-C) and Lateral (D-F) views with safe needle advancement with a superior lateral transpedicular approach heading in an inferior medial direction. Notes: Needle point at lateral border (A), posterior aspect of pedicle (D), mid-pedicle (B and $\mathbf{E})$, medial border $(\mathbf{C})$, and posterior wall of vertebra $(\mathbf{F})$. Image courtesy of Relievant Medsystems Inc.

\section{BVN RFA Impacts on Functional Outcomes}

Most studies reviewed reported ODI as the tool of choice to measure functional outcomes at multiple follow-up periods, which were variable per each individual study. ${ }^{6-10,12-14,30,31}$ Becker et al (2017) concluded that BVN RFA significantly improved functional outcomes for up to 1-year during the study period, as measured by ODI. ${ }^{13}$ The primary endpoint of ODI at 3-month follow-up demonstrated statistically significant 
therapeutic benefit, reducing the ODI from 52 preintervention to 23 post-intervention $(\mathrm{p}<0.001)$. Moreover, this benefit was maintained with regards to ODI at 6 weeks, 3 months, 6 months, and 12 months. The overall patient treatment success rate using the study-defined criteria at 12 months was 13/16 patients or $81 \%$. The three patients not meeting the combined study success criteria failed to exhibit meaningful clinical improvement, as defined as at least a 10-point ODI improvement. $^{13}$ Few conclusions can be drawn from this study beyond that BVN RFA appears to have demonstrated improvement in the studied cohort as measured by ODI from 3 to 12 months post-procedure. However, without a control group, there exists the possibility that this would have occurred without this intervention. Fischgrund et al (2018) demonstrated very similar outcomes, with an average decrease in ODI of $>20$ points, suggesting that BVN RFA is clinically effective in patients with CLBP associated with MCs type I or II, with reported scores improved at both 2 and 6 weeks and at 3, 6, and 12 months. ${ }^{9}$ Commendably, this study introduced a sham procedure, which was identical to the intervention procedure without ever turning the RF device on. However, it is worth noting that a placebo effect to device trials is known and could have played a role here. ${ }^{32}$ Truumees et al (2019) demonstrated that $93 \%$ and $75 \%$ of patients had a $>10$-point and $>20$-point ODI improvement, respectively after 3 months $(p<0.0001)$. The average ODI score of patients in the treatment group decreased from 48.5 pre-treatment to 18.4 post-treatment at 3-month follow-up, a difference of $>30$ points, suggesting significant, meaningful clinical improvement, which was in line with similar previously reported studies. ${ }^{7}$ Khalil et al (2019) reported $74.5 \%$ of patients of the BVN RFA group had an ODI improvement of $>10$ points compared to only $32.7 \%$ in the standard of care group at 3-month follow-up. ${ }^{8}$

Similar functional clinical improvement was also reported by Fischgrund et al (2019) in a study reporting a significant reduction in ODI, which improved by a mean of $53.7 \%$ ( $<<0.001$ ), at the final follow-up at 24 months. ${ }^{6}$ The study concluded that BVN RFA demonstrated sustained clinical benefits in ODI for the treatment arm at 2-year post-treatment follow-up, and that BVN RFA is a reliable, durable treatment for vertebrogenic pain. Kim et al (2020) reported that BVN RFA for CLBP with type I and II MCs demonstrated ODI improvement from preoperative state at 1 week, 6 months, and final follow-up were $45.8 \pm 8.7,50.4 \pm 8.2$, and $52.7 \pm 10.3$, respectively $(p<0.0001){ }^{31}$ The study by Markman et al (2020) reported that there is an association between functional improvement (ODI) following BVN RFA and lower dose opioid utilization. ${ }^{10}$ However, since the primary outcome of the study was not ODI changes but, rather, to report the association between opioid utilization and other outcome measurements (ODI, VAS), this will be described in detail later in this review. Longer follow-up data from the Fischgrund et al (2020) study also noted significant improvement in ODI at five years post-intervention, with up to $65 \%$ of patients returning to their prior level of activity and $77 \%$ of patients having a $>15$ point improvement in ODI at five-year follow-up. ${ }^{12}$ More recently, De Vivo et al (2020) reported supportive findings to all of the above-cited studies, documenting ODI improvements at 3-month and 12-month follow-ups and reporting a mean change of -32.4 points (range was -6 to -42 ). Clinical success, defined as $\geq 10$ points reduction in ODI, was achieved in $96.5 \%$ of patients $(54 / 56){ }^{30}$

Our current literature review of available up-to-date publications (as of March 2021) utilizing BVN RFA in the treatment of vertebrogenic pain suggests that there is an agreement amongst all studies reporting statistically significant and meaningful clinical improvement in functional outcomes at 3-month follow-up, with a single study supporting efficacy at up to 5 years postoperative followup. While no formalized evidentiary analysis was performed, our narrative review agrees with the conclusion of Conger et al that there exists moderate-quality evidence that BVN RFA is an effective intervention for reduction of disability and improvement in function, as measured by the ODI at short- and long-term follow-up for patients

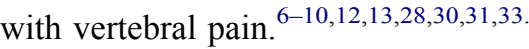

\section{BVN RFA Impacts on Pain Perception}

The visual analog scale (VAS) was the outcome tool utilized to measure pain perception by all clinical studies that assessed changes in pain outcomes. ${ }^{6-10,12-14,30,31}$ In the study by Becker et al (2017), although VAS was not the primary endpoint, the authors reported an improvement in VAS from $61 \pm 22$ pre-operative (baseline) to $38 \pm 30$ and $45 \pm 35$ at 6-week and 3-month follow-ups, respectively, which were statistically significant findings $(p<0.05)$. However, this study lacked a control group and, without a control arm, it is difficult to determine whether these improvements were the results of regression to the mean, natural disease progression, device placebo effect, or an alternative intervention not captured in the study. The authors concluded that BVN ablation significantly 
improved VAS patient-reported outcomes, which were sustained for up to 3-months. ${ }^{13}$ In a study by Kim et al (2018), the VAS was used as the primary endpoint at 3 months to assess pain, and this study also uniquely used a transforaminal epiduroscopic laser ablation approach. In this retrospective study, preoperative VAS scores of $7.79 \pm$ 0.97 decreased to $2.21 \pm 0.89$ at the 3 -month follow-up (p $<0.0001)$. This study was particularly interesting because it included patients who had a history of spinal surgery, which allows the sample to be slightly more representative of the typical patient in a spine population. However, the lack of a control arm ultimately makes the results of the study difficult to assess whether the results were due to the BVN RFA, VAS regression to the mean, natural regression of symptoms of the disease course, or an alternative intervention either not described or beyond the scope of the manuscript. At a mean follow-up of 15 months, VAS was reduced to $2.36 \pm 1.01$ from the preoperative baseline. The authors concluded that transforaminal BVN RFA was a promising treatment option to reduce pain intensity measured by VAS in patients with vertebrogenic pain accompanied by Modic changes on MRI. ${ }^{14}$ In 2019, a BVN RFA study by Truumees et al had VAS as a secondary endpoint and reported a mean baseline VAS of $6.36 \pm 1.02$ that improved to a mean VAS of $2.86 \pm 2.25$ at the 3-month post-BVN RFA follow-up and $1.42 \pm 1.77$ at the 6-month follow-up $(\mathrm{p}<0.0001)$. With regards to the sample population, this study included, patients with vertebrogenic pain and MC type 1 and type 2 on MRI, and allowed patients with asymptomatic spinal stenosis, a history of lumbar spine surgery (without hardware remaining) if greater than 6 months prior to study inclusion, and patients who used extended-release narcotics. By including patients with moderate spinal stenosis, on extended-release opiates, and with a history of lumbar surgery the sample, although small, does approach a more generalizable population, which is arguably more representative of our spine population in daily clinical practice. $^{7}$

The clinical studies by Fischgrund et al ${ }^{6,9,12}$ have produced perhaps the most ambitious BVN RFA literature to date. The Surgical Multi-Center Assessment of RF Ablation for the Treatment of Vertebrogenic Back Pain (SMART) study collected data from October 2011 to March 2016. Iit reported outcome data in 2018, 2019, and 2020 regarding 3-month, 6-month, 1-year, 2-year, and 5-year follow-ups. In contrast to some, but not all, of the previous studies, this study excluded those patients with a history of lumbar surgery, spinal stenosis with neurogenic claudication, radicular pain, $>5 \mathrm{~mm}$ disc extrusion or protrusion, osteoporosis, spondylolisthesis $>2 \mathrm{~mm}$ at any spinal level, $>3$ Waddell signs (which suggest potential non-organic/psychological components to CLBP), $>24$ on the Beck Depression Inventory, current use of extended-release narcotics, those receiving disability payments, or those currently involved in litigation related to CLBP. The inclusion and exclusion criteria for this study is worth discussing as, while the cohort sample is well defined, many of the exclusion criteria are commonly found amongst patients in a spine clinic, which would potentially make the study population less generalizable in our opinion. The robust data from these studies focused on the specific population of chronic vertebrogenic pain $>6$ months duration who have failed conservative management and with evidence of type 1 or type 2 MCs on MRI, in which BVN RFA has been continuously endorsed as statistically and clinically effective.

In Fischgrund et al (2018), with regards to VAS, at 3 months there was a non-statistically significant $(p=0.083)$ difference between the per-protocol treatment arm and the sham arm. However, at 6 months and 12 months, the per protocol treatment arm demonstrated a statistically significant improvement ( $\mathrm{p}=0.008$ and 0.038 , respectively) when compared to the sham arm. At 6-months, VAS was $3.74 \pm$ 2.68 vs $4.41 \pm 2.76$ for the sham arm, and at 12 months VAS was $3.96 \pm 2.83$ vs $4.46 \pm 2.78$ for the sham arm. ${ }^{9}$ At 1 year, per the protocol, patients in the sham arm were offered the ability to crossover into the treatment arm, and a majority $(73 \%, \mathrm{n}=54)$ of them decided to do so. Given a lack of patients remaining in the sham sample there was an inability to draw conclusions from an inadequate sample, which lacked statistical power. At the 1-year mark, this study transitioned into a single-arm, unblinded, prospective trial. Of the original 128 patients in the per protocol treatment arm, 104 were present at the 2-year follow-up and reported a mean VAS of $3.13 \pm 2.64$ compared to a baseline of $6.73 \pm 1.38$. All changes in VAS from preoperative baseline to postoperative VAS at 3, 6, 12, and 24 months were statistically significant with $\mathrm{p}<0.001$ in these patients with a 24-month follow-up VAS. ${ }^{6}$

At this point, the main takeaway we want to highlight to spine physicians is that based on this cumulative data, patients meeting this study's rigid selection criteria may expect a nearly 50\% reduction in VAS at 24-month followup. Furthermore, at 5 years, 100 of the 117 (85\% retention) per protocol patients in the randomized treatment arm who 
reported VAS results demonstrated a reduction of 4.38 \pm 2.35 in mean VAS from an original preoperative mean VAS baseline of $6.74(p<0.001)$. Using an established minimum clinically important difference (MCID) of 2 points for the VAS, $88 \%$ of patients enrolled achieved the goal of MCID in pain perception at 5-year follow-up postoperatively. $^{12}$

In 2019, Khalil et al conducted a prospective, openlabel, multi-center, randomized controlled trial comparing the effectiveness of BVN RFA to standard of care treatment of vertebrogenic pain. Again, this study criteria included 140 patients with type 1 and/or type 2 MCs on MRI, which is phenotypically suggestive of vertebrogenic pain syndrome, however it excluded patients with symptomatic spinal stenosis, radicular symptoms, disc protrusions $>5 \mathrm{~mm}$, spondylolisthesis $>2 \mathrm{~mm}$ at any level, or a Beck's Depression Index $>24$. Of note, the standard of care treatment arm included: medications, therapy, manipulation, acupuncture, and spinal injections. At 3 months, the secondary endpoint of VAS demonstrated a statistically significant improvement $(\mathrm{p}<0.001)$ from $6.51 \pm 1.31$ in the treatment arm to $3.11 \pm 2.64$, while the standard of care arm baseline 6.82 \pm 1.34 improved to $5.73 \pm 2.16^{8}{ }^{8}$

In 2020, Kim et al published a prospective case series evaluating BVN RFA and its impacts on pain perception measured by VAS at 1 week, 6-month, and 12-month follow-up. VAS showed a statistically significant $(p<0.0001)$ improvement from a preoperative baseline of 7.37 which decreased to 2.96 at 1-week follow-up, 1.97 at 6-month follow-up, and 1.67 as a final follow-up postoperatively. However, one caveat was that it was difficult to establish when exactly the final follow-up was for each patient, as the reported mean follow-up duration was 14.9 months. In the same year of 2020, Markman et al randomized a BVN RFA group and a sham group assessing multiple outcomes, including VAS and the association of opioid utilization; ${ }^{31}$ however, since opioid utilization and not VAS was the primary outcome, this study will be discussed in detail later in this review. De Vivo et al (2020) reported that targeted BVN RFA was considered clinically successful in most patients $(54 / 56)$, based on 12 month VAS follow-up data. Clinical success was defined as at least a 2.0-point reduction in VAS and a 10-point reduction in ODI (the beforehand mentioned minimum clinically important difference). At 12-months follow-up, VAS decreased significantly compared to baseline, and the mean decrease was $4.3 \mathrm{~cm}$, with 54 out of 56 patients
(96.4\%) reporting a VAS decrease of at least $3.0 \mathrm{~cm}$ (greater than the MCID). ${ }^{30}$ It is noteworthy that 5 -year long-term follow-up data from Fischgrund et al (2020) reported that very few $(4 \%)$ patients received spinal interventions (such as epidural steroid injections) in the preceding five-years, suggesting that pain was substantially controlled without the need of other spinal interventions at 5-year follow-up postoperatively from the BVN RFA. This is quite remarkable, considering that a great number of patients (up to $70 \%$ ) had CLBP at baseline for more than five years and had received at least one injection within that timeframe and had failed conservative treatments for CLBP. $^{12}$

While there is limited data from less than a dozen studies, for spine physicians the cumulative data described above may be the best evidence to date to show superiority of BVN RFA, compared to the current standard of care for vertebral pain associated with Modic changes. Our review findings are in agreement with other studies that based on the current literature there is limited moderate-quality evidence that BVN RFA is superior to conservative care for pain reduction, at least at 3-month follow-up, if not longer, as measured by the VAS documented by the numerous studies statistically analyzed and highlighted above. $^{6,8,9,12,28,30,33}$

\section{BVN RFA Impacts on Quality of Life}

A few studies in the current literature report quality of life (QoL) outcome measurements pre and post BVN ablation intervention. The two measurements utilized were the SF36 (physical and mental components) and the EQ-5D-5L questionnaire. The minimal clinically important difference (MCID) for these two measures has been published at 4.9-point increase for SF-36 physical component score (PCS) and 0.03-point improvement for EQ-5D-5L. ${ }^{34,35}$ In 2017 Becker et al reported a preoperative mean of $34.5 \pm 6.5$ on SF-36 PCS with an increase of 7.2 points to 41.7 at 3 months follow-up, which was statistically significant $(p=0.03) .{ }^{13}$ In contrast, Fischgrund et al 2018 demonstrated different results, suggesting that SF-36 PCS component changes were not significant $(p>0.05)$ at 3,6 , and 12 months when comparing the treatment-arm to the shamarm. Likewise, mental component changes were not significant between arms at 3 and 6 months, however, patients did report significant differences at 12 months $(\mathrm{p}<0.021) .{ }^{9}$ In treatment arm patients with 24 months of follow-up, paired $t$-test intra-patient differences of SF-36 PCS were significant to baseline at each timepoint of follow-up. Preoperative 
baseline mean SF-36 PCS was $33.5 \pm 7.5$, and changed to a postoperative score of $43.3 \pm 9.5$ at 3 -months, $43.9 \pm 8.7$ at 6-months, $42.8 \pm 9.2$ at 12-months and $45.8 \pm 9.2$ at 24months postoperatively, all values were found to be statistically significant $(\mathrm{p}<0.001){ }^{6}$ However, these results were not compared to the sham-arm beyond 12 months, as both groups were unblinded upon offering the sham-arm crossover. As $73 \%$ of the sham group elected for crossover, the authors deemed the sham population to be too small to compare, due to inadequate statistical power. In the same year, Khalil et al 2019 reported significant differences in both physical and mental component SF-36 and EQ-5D5L scores between BVN RFA and standard of care treatment for vertebral pain at 3-months of follow-up. The least square change from baseline between the BVN RFA and standard of care arm was 2.615 versus -2.786 for the SF-36 (mental component) a difference of 5.041 between arms, and 14.021 versus 2.114 for the SF-36 (physical component) a between arm difference of 11.907. The authors concluded that the difference between the two arms adjusted means was statistically significant $(\mathrm{p}<0.001)$. The same study also analyzed changes in the EQ-5D-5L questionnaire between the two arms and least square mean change from baseline was 0.1803 in the treatment group and 0.0135 in the standard of care group, resulting in an adjusted mean difference statistically significant between the two arms of $0.1668(p<0.001){ }^{8}$ Supporting the abovedescribed findings, Truumees et al 2019 published an openlabel, single-arm case series study on BVN RFA and measured quality of life reported outcomes at 3-months post-procedure. The study reported a significant improvement in EQ-5D-5L (0.198 \pm 0.129$)$ and SF-36 from 31.62 at baseline to 47.41 at 3-month for the physical component, and preoperative 51.01 and 55.24 at 3-month follow-up for the mental component of SF-36, similar to previous studies that were statistically significant $(\mathrm{p}<0.0001) .^{7}$

Although only five studies reported QoL outcome measurements, the cumulative data among all studies is very promising suggesting significant improvement in QoL, most commonly measured by the SF-36 form. Results were not unanimous, as one study did not find statistically significant findings, however pooled data from the other studies was statistically significant. SF-36 was not significantly different when compared to treatment vs sham arm at 12-months follow-up, ${ }^{9}$ however in the open-label treatment arm follow-up study data at 24-months, QoL improvement from baseline in paired analysis was statistically significant at each follow-up timepoint. ${ }^{6}$ Our current literature suggests that there is limited evidence that BVN RFA is an effective intervention for improvement in QoL. ${ }^{6-9,13}$

\section{BVN RFA Impacts on Opioid Utilization}

Chronic opioid therapy is an ongoing problem in the United States healthcare system, and opioids are the most commonly prescribed medication for patients with CLBP. $^{36}$ Multiple studies have described an association between decreased opioid utilization after spinal interventions, ${ }^{37,38}$ as well as an inversely related relationship, in which patients on chronic opioid therapy, prior to spinal interventions may respond in a different manner to spinal interventions, when compared to lower-dose or opioid-naive patients. ${ }^{39,40}$ Therefore, the appropriate use of opioid medication is an important consideration in patients undergoing BVN ablation, and a very limited number of studies have looked into the impact of BVN ablation on opioid utilization, or vice-versa.

In 2019, the studies by Truumees et al, Khalil et al and Fischgrund et al reported opioid utilization as a secondary outcome measurement. ${ }^{6-8}$ Truumees et al noted that $25 \%$ of patients undergoing BVN RFA were using opioids at baseline. Of these, $50 \%$ discontinued opioid use at the 3-month follow-up post procedure and none increased their dose or required new opioid medication. ${ }^{7}$ In contrast, Khalil et al reported that $32 \%$ of patients on their study used opioids at baseline, and no significant difference was observed between the two treatment arms at 3-months. ${ }^{8}$ Fischgrund et al reported that of the 106 patients in the 2-year follow-up group, 28 had been taking opiates at baseline. At 12 months follow-up, 46.4\% had completely stopped opiate use and an additional 14.3\% had reduced their opiate usage from their baseline. ${ }^{6}$

In 2020, the studies by Fischgrund et al and Markman et al provided additional data regarding the impact of BVN RFA on opioid utilization at long-term follow-up and as a primary outcome. ${ }^{10,12}$ Fischgrund et al five-year follow-up data reported that 34 of the 100 patients $(34 \%)$ that matriculated to the final follow-up had been taking opiates at baseline, 22 of these patients had completely stopped opiate use by the five-year follow-up. In the remaining 12 patients taking opiates at baseline, 8 were actively using opiates (more than $25 \%$ of the time) and 4 reported taking opiates less than a single time per week. ${ }^{12}$ The study by Markman et al (2020) is the only study to date that solely focuses on opiate use correlated with in function and pain as a primary outcome in the BVN RFA population. Using data 
from the Fischgrund 2018 study, they demonstrated that at 12 months follow-up from BVN RFA, $60 \%$ of opiate users in the treatment arm reduced opiate usage, compared to $40 \%$ who increased their usage. Functional improvement differences at 12 months were significant between baseline opiate users who reduced their opiate use post BVN RFA with a mean ODI reduction of $-24.9 \pm 16.0$, compared to those who increased their opiate use post treatment, with a mean ODI reduction of $-7.3 \pm 9.8(\mathrm{p}<0.001)$. In contrast, the sham arm did not demonstrate significant differences in functional improvements between the $78 \%$ of patients that reduced their opiate use at a mean ODI reduction of $-17.4 \pm 16.1$, and the $22 \%$ of opiate users who increased their usage from baseline at a mean ODI reduction of $-1.2 \pm 14.3(p=0.53)$. Additionally, the study noted that in the treatment arm, patients who had a decrease of VAS (by 33mm) at 12months follow-up, also had a concomitant decrease in opioid utilization. In contrast to patients that had an increase in opioid use during the study at 12-month follow-up, these only had a not clinically significant reduction in VAS of $0.6 \mathrm{~mm}$. This study demonstrated that the pain and functional improvements were correlated with the treatment by ablation rather than the increase in opiate use. ${ }^{10}$

These studies combine to show what appears to be, at a minimum, a reduction in opiate usage associated with successful BVN RFA treatment. However, the only aforementioned study by Markman et al which had a control group found no statistically significant change in opiate usage between treatment and control. While the other studies found a clear decrease in usage, it is challenging to affirm the benefit of BVN RFA on opiate use from a statistical standpoint; however, based on the studies and data reviewed, there is at least an associated decline in opiate use with successful BVN RFA.

\section{BVN RFA Impacts on Patient Satisfaction}

Two studies looked at post-procedure patient satisfaction, as measured by MacNab's criteria, at one week and at an undefined time. The MacNab criteria is a well-known outcome assessment of patient satisfaction used in the scientific spine community, in which the patient is asked to rate their level of well-being, generally after the surgical procedure, choosing amongst four options: excellent, good, fair, and poor. The MacNab criterion is established as an acceptable outcome to describe satisfaction and success; however, it should not be used in isolation and must be used in conjunction with another outcome to support its findings.
In the Truumees et al 2019 study, $>78 \%$ of subjects enrolled considered the treatment with BVN RFA a success to improve their pain and function, and were satisfied with their outcomes, however this was subjectively reported and not using the MacNab criteria. ${ }^{7}$ Kim et al 2018 assessed the MacNab criteria at one-week follow-up, while the study by Kim et al 2020 assessed $\mathrm{MacNab}$ criteria at an indeterminate length of time post procedure. The 2018 study revealed a success rate of $92.9 \%$ at 1 -week follow up and the 2020 study revealed a 93\% success rate (good to excellent satisfaction) at unspecified follow-up periods. Impressively, none of the patients across both studies reported their post-procedure satisfaction as poor, defined per MacNab Criteria as no improvement or insufficient improvement to increase activities. $^{14,31}$

\section{Safety Profile}

There were no device-related patient deaths or serious adverse events (AEs) based on the available published literature. However, only a few studies discussed safety implications. Specifically, there were no reports of spinal cord injuries, thermal injuries, avascular necrosis, pedicle fractures, infections, or broken devices. AEs were defined as procedure-related: incisional pain, urinary retention, meralgia paresthetica, new-onset back pain, or new-onset

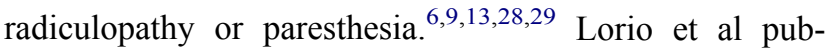
lished a guideline regarding BVN RFA for vertebrogenic pain compiling multiple studies and suggested that BVN RFA for a specific subset of vertebrogenic pain patients with MCs on MRI has an excellent safety profile, when compared to other interventions. ${ }^{1}$ A systematic review of BVN ablation agreed. It reported adverse events were rare in all studies reviewed. ${ }^{33}$ The most severe AEs reported included: persistent radiculopathy was noted in one case with potential pedicle tract issues, one vertebral compression fracture in an osteoporotic patient on high dose hormonal therapy, and one case of retroperitoneal hemorrhage due to misdirected pedicle access. While this appears to be safe intervention, potential complications may arise from inappropriate needle placement, which may lead to nerve root injury or hematoma; hence, the importance of frequent fluoroscopy views checks as the introducer is advanced. ${ }^{29}$ Few studies published in the current literature discuss the safety of BVN RF in the treatment of CLBP, which is a limitation of our review. There was a low rate and severity of device and/or procedure-related AEs, and the most common AEs noted were mild incisional pain 
and leg paresthesia. General contra-indications to the procedure itself regardless of patient selection criteria include active systemic infections, pregnancy, skeletal immaturity, prior fusion at the treatment level, type $3 \mathrm{MCs}$, and implantable pulse generators. ${ }^{9,29}$

Device-procedure related AEs are very low. Among all 473 clinical trials BVN RFA procedures, including 78 sham procedures, the following AEs have been reported: 2 radiculopathy cases, 1 nerve root injury, 4 transient motor/sensory deficits, 17 transient radiculitis cases treated orally with medication, 1 retroperitoneal hemorrhage and 1 vertebral compression fracture. Nonetheless, based on the studies available, BVN ablation seems to be safe and well tolerated by most patients when proper patient selection and procedural technique are applied.

\section{Limitations}

Per the constraints of our review, there are only 15 studies published to date regarding the topic. Among these, two were directly supported by industry funding, which increases the risk of publication bias.

Our study is a review of the literature following the PRISMA guidelines and summarizing a quality appraisal table of published literature, including RCTs, systematic reviews, observational studies and narrative reviews. From a statistical standpoint, this study reviews each findings from each study published to date and descriptively describes these statistics in detail in Table 1, including primary outcomes, estimates, sample size, authors, publication year/journal, follow-up, etc. Although our methodology followed the PRISMA guidelines, our study has limitations and is not a systematic review. Again, there were only 15 studies to review, which satisfied our inclusion and exclusion criteria. It is always prudent to comment on the limitations of generalizability in such a setting. Additionally, our review evaluated three different technical approaches, by many different physicians, from different backgrounds, with presumably differing amounts of experience with the procedure itself. Thus, a high level of heterogeneity is introduced.

Most importantly, the only commentary that can truly be pulled from this review is subject to the selection criteria from which the patients were selected in each study. While BVN RFA may prove to be a successful treatment, we can only comment on its success as it relates to the rigid selection criteria upon which the foundational studies were completed. Any extrapolation of these findings to a differing patient population should be considered experimental and not supported by the data discussed in this review. Again, we must urge readers to be cognizant that while there has yet to be a study published to date on the amount of patients who may qualify for BVN RFA via the selection criteria described in the Fischgrund or Khalil studies in general practice, but there is an inherent understanding among spine providers that the percent of patients who meet such a rigid criteria for treatment is likely low in clinical practice.

\section{Conclusion}

The current literature demonstrates consistent positive outcomes across multiple studies that basivertebral nerve ablation is effective in reducing pain and disability in appropriately selected patients with chronic axial low back pain of vertebrogenic nature at a minimum of three months follow-up, but perhaps much longer. Furthermore, there is evidence that this intervention is more beneficial than the current standard of care for the treatment of vertebrogenic pain. However, additional non-industry funded high-quality research and perhaps a more generalizable patient population is needed to confirm these findings.

Our review concluded that there is a clinical and statistically significant treatment effect of basivertebral nerve ablation for vertebrogenic pain with type 1 or 2 Modic changes in pain reduction, functional improvements, opioid dose reduction, and improved quality of life. There were no reported device-related patients deaths, and few serious adverse events based on the available literature. Basivertebral nerve ablation appears to be a safe and efficacious intervention for vertebrogenic pain, when proper patient selection and procedural techniques are applied.

\section{Abbreviations}

BVN, basivertebral nerve; RFA, radiofrequency ablation; CLBP, chronic low back pain; ODI, Oswestry Disability Index; VAS, visual analog scale; SF-36, short form 36; AEs, adverse events; MRI, magnetic resonance images; MCs, Modic changes; IVD, Intervertebral Disc; MCID, Minimum Clinically Important Difference.

\section{Acknowledgments}

The authors would like to acknowledge and thank Conan So (in memoriam), David Schulze and William Ramos for their contributions to the primordial works of this study. Also, we would like to thank Douglas Beall MD, and Lynn Chollet Hinton PhD, MSPH for their statistical and quality content review of this manuscript. 


\section{Author Contributions}

All authors made a significant contribution to the work reported, whether that is in the conception, study design, execution, acquisition of data, analysis and interpretation, or in all these areas; took part in drafting, revising or critically reviewing the article; gave final approval of the version to be published; have agreed on the journal to which the article has been submitted; and agree to be accountable for all aspects of the work.

\section{Funding}

This project was supported by an unrestricted grant from Relievant to the American Society of Pain and Neuroscience.

\section{Disclosure}

The authors have no conflicts of interest related to this study.

\section{References}

1. Lorio M, Clerk-Lamalice O, Beall DP, et al. International society for the advancement of spine surgery guideline - intraosseous ablation of the basivertebral nerve for the relief of chronic low back pain. Int J Spine Surg. 2020;14(1):18-25. doi:10.14444/7002

2. Deyo RA, Mirza SK, Martin BI. Back pain prevalence and visit rates. Spine. 2006;31(23):2724-2727. doi:10.1097/01.brs.0000244618. j06877.cd

3. Frymoyer JW. Back pain and sciatica. $N$ Engl J Med. 1988;318 (5):291-300. doi:10.1056/NEJM198802043180506

4. Hoy D, Bain C, Williams G, et al. A systematic review of the global prevalence of low back pain. Arthritis Rheum. 2012;64(6): 2028-2037. doi:10.1002/art.34347

5. Dagenais S, Caro J, Haldeman S. A systematic review of low back pain cost of illness studies in the United States and internationally. Value Health. 2008;11(3):A160-A161. doi:10.1016/S1098-3015(10) 70509-X

6. Fischgrund JS, Rhyne A, Franke J, et al. Intraosseous basivertebral nerve ablation for the treatment of chronic low back pain: 2-year results from a prospective randomized double-blind sham-controlled multicenter study. Int J Spine Surg. 2019;13(2):110-119. doi:10.14 444/6015

7. Truumees E, Macadaeg K, Pena E, et al. A prospective, open-label, single-arm, multi-center study of intraosseous basivertebral nerve ablation for the treatment of chronic low back pain. Eur Spine J. 2019;28(7):1594-1602. doi:10.1007/s00586-019-05995-2

8. Khalil JG, Smuck M, Koreckij T, et al. A prospective, randomized, multicenter study of intraosseous basivertebral nerve ablation for the treatment of chronic low back pain. Spine J. 2019;19(10):1620-1632. doi:10.1016/j.spinee.2019.05.598

9. Fischgrund JS, Rhyne A, Franke J, et al. Intraosseous basivertebral nerve ablation for the treatment of chronic low back pain: a Prospective Randomized Double-Blind Sham-Controlled Multi-Center Study. Eur Spine J. 2018;27(5):1146-1156. doi:10.10 07/s00586-018-5496-1

10. Markman JD, Rhyne AL, Sasso RC, et al. Association between opioid use and patient-reported outcomes in a randomized trial evaluating basivertebral nerve ablation for the relief of chronic low back pain. Neurosurgery. 2020;86(3):343-347. doi:10.1093/neuros/nyz093
11. Deyo RA, Weinstein JN. Low back pain. $N$ Engl J Med. 2001;344 (5):363-370. doi:10.1056/NEJM200102013440508

12. Fischgrund JS, Rhyne A, Macadaeg K, et al. Long-term outcomes following intraosseous basivertebral nerve ablation for the treatment of chronic low back pain: 5-year treatment arm results from a prospective randomized double-blind sham-controlled multi-center study. Eur Spine J. 2020;29(8):1925-1934. doi:10.1007/s00586-02006448-X

13. Becker S, Hadjipavlou A, Heggeness MH. Ablation of the basivertebral nerve for treatment of back pain: a clinical study. Spine J. 2017;17(2):218-223. doi:10.1016/j.spinee.2016.08.032

14. Kim HS, Adsul N, Yudoyono F, et al. Transforaminal epiduroscopic basivertebral nerve laser ablation for chronic low back pain associated with modic changes: a Preliminary Open-Label Study. Pain Res Manag. 2018;2018:6857983. doi:10.1155/2018/6857983

15. Modic MT, Steinberg PM, Ross JS, et al. Degenerative disk disease: assessment of changes in vertebral body marrow with MR imaging. Radiology. 1988;166(1):193-199. doi:10.1148/radiology.166.1.3336 678

16. Fras C, Kravetz P, Mody DR, et al. Substance P containing nerves within the human vertebral body: an immunohistochemical study of the basivertebral nerve. Spine J. 2003;3(1):63-67. doi:10.1016/ S1529-9430(02)00455-2

17. Kjaer P, Korsholm L, Benedix T, et al. Modic changes and their association with clinical findings. Eur Spine J. 2006;15 (9):1312-1319. doi:10.1007/s00586-006-0185-x

18. Jensen RK, Leboeuf-Yde C. Is the presence of Modic changes associated with the outcomes of different treatments? A systematic critical review. BMC Musculoskelet Disord. 2011;12(1):183. doi:10.11 86/1471-2474-12-183

19. Bailey JF, Liebenberg E, Degmetch S, et al. Innervation patterns of PGP 9.6-positive nerve fibers within the human lumbar vertebra. J Anat. 2011;218(3):263-270. doi:10.1111/j.1469-7580.2010.01332.x

20. DePalma MJ, Ketchum JM, Saullo T. What is the source of chronic low back pain and does age play a role? Pain Med. 2011;12 (2):224-233. doi:10.1111/j.1526-4637.2010.01045.x

21. Lotz JC, Fields AJ, Liebenberg EC. The role of the vertebral endplate in low back pain. Glob Spine J. 2013;3(3):153-163. doi:10.1055/ s-0033-1347298

22. Kaplan KM, Spivak JM, Bendo JA. Embryology of the spine and associated congenital anomalies. Spine J. 2005;5(5):563-576. doi:10.1016/j.spinee.2004.10.044

23. Tzika M, Paraskevas GK, Piagkou M, et al. Basivertebral foramen of true vertebrae: morphology, topography and clinical considerations. Surg Radiol Anat. 2021. doi:10.1007/s00276-021-02690-0

24. Weishaupt D, Zanett M, Hodler J, et al. Painful lumbar disc derangement: relevance of endplate abnormalities at MRI imaging. Radiology. 2001;218(2):420-427. doi:10.1148/radiology.218.2.r01fe 15420

25. Carriage EJ, Alamin TF, Miller JL, et al. Discography, MRI and psychosocial determinants of low back pain disability and remission: a prospective study in subjects with benign persistent back pain. Spine J. 2005;5(1):24-35. doi:10.1016/j.spinee.2004.05.250

26. Kuisma M, Karppinen J, Niinimak J, et al. Modic changes in endplates of lumbar vertebral bodies: prevalence and association with low back pain among middle-aged male workers. Spine. 2007;32 (10):1116-1122. doi:10.1097/01.brs.0000261561.12944.ff

27. Mok K, Samartzis D, Karppinen J, et al. Modic changes of the lumbar spine: prevalence, risk factor, and association with disc degeneration and low back pain in a large-scale population based cohort. Spine J. 2016;16(1):32-41. doi:10.1016/j.spinee.2015.09.060

28. Urits I, Noor N, Johal AS, et al. Basivertebral nerve ablation for the treatment of vertebrogenic pain. Pain Ther. 2020.

29. Nguyen KML, Nguyen DTD. Minimally invasive treatment for degenerative lumbar spine. Tech Vasc Interv Radiol. 2020;23 (4):100700. doi:10.1016/j.tvir.2020.100700 
30. De Vivo AE, D'Agostino G, D'Anna G, et al. Intraosseous basivertebral nerve radiofrequency ablation (BVA) for the treatment of vertebrogenic chronic low back pan. Neuroradiology. 2020.

31. Kim HS, Wu PH, Jang I. Lumbar degenerative disease part 1: anatomy and pathophysiology of intervertebral discogenic pain and radiofrequency ablation of basivertebral and sinuvertebral nerve treatment for chronic discogenic back pain: a prospective case series and review of literature. Int J Mol Sci. 2020;21(4):1483.

32. Koshi EB, Short CA. Placebo theory and its implications for research and clinical practice: a review of the recent literature. Pain Pract. 2007;7(1):4-20. doi:10.1111/j.1533-2500.2007.00104.x

33. Conger A, Schuster NM, Cheng DS, et al. The effectiveness of intraosseous basivertebral nerve radiofrequency neurotomy for the treatment of chronic low back pain in patients with Modic changes: a systematic review. Pain Med. 2021;22(5):1039-1054. doi:10.1093/ $\mathrm{pm} / \mathrm{pnab} 040$

34. Copay AG, Glassman SD, Subach BR, et al. Minimum clinically important difference in lumbar spine surgery patients: a choice of methods using the Oswestry disability index, medical outcomes study questionnaire short form 36, and pain scales. Spine J. 2008;8 (6):968-974. doi:10.1016/j.spinee.2007.11.006

35. Herdman M, Gudex C, Lloyd A, et al. Development and preliminary testing of the new five-level version of EQ-5D (EQ-5D-5L). Qual Life Res. 2011;20:1727-1736.
36. Deyo RA, Smith DHM, Johnson ES, et al. Opioids for back pain patients: primary care prescribing patterns and use of services. J Am Board Fam Med. 2011;24(6):717-727. doi:10.3122/jabfm.2011.06.100232

37. Al-Kaisy A, Van Buyten JP, Amirdelfan K, et al. Opioid-sparing effects of $10 \mathrm{~Hz}$ spinal cord stimulation: a review of clinical evidence. Ann N Y Acad Sci. 2020;1462(1):53-64. doi:10.1111/nyas.14236

38. Al-Kaisy A, Van Buyten JP, Carganillo R, et al. $10 \mathrm{kHz}$ SCS therapy for chronic pain, effects on opioid usage: post hoc analysis of data from two prospective studies. Sci Rep. 2019;9(1):11441. doi:10.1038/ s41598-019-47792-3

39. Kirpalani D, Mitra R. Is chronic opioid use a negative predictive factor for response to cervical epidural steroid injection? J Back Musculoskelet Rehabil. 2011;24(3):123-127. doi:10.3233/BMR-2011-0285

40. Sharan AD, Riley J, Falowski S, et al. Association of opioid usage with spinal cord stimulation outcomes. Pain Med. 2018;19 (4):669-707. doi:10.1093/pm/pnx262

41. Page MJ, McKenzie JE, Bossuyt PM, et al. The PRISMA 2020 statement: an updated guideline for reporting systematic reviews. BMJ. 2021;372:n71. doi:10.1136/bmj.n71

\section{Publish your work in this journal}

The Journal of Pain Research is an international, peer reviewed, open access, online journal that welcomes laboratory and clinical findings in the fields of pain research and the prevention and management of pain. Original research, reviews, symposium reports, hypothesis formation and commentaries are all considered for publication. The manuscript management system is completely online and includes a very quick and fair peer-review system, which is all easy to use. Visit http:// www.dovepress.com/testimonials.php to read real quotes from published authors. 